\title{
Nobel Lecture: Controlling photons in a box and exploring the quantum to classical boundary"
}

\author{
Serge Haroche \\ Laboratoire Kastler Brossel de l'Ecole Normale Supérieure, 24 Rue Lhomond, 75231, Paris, \\ and Collège de France, 11 Place Marcelin Berthelot, 75005, Paris, France
}

(published 12 July 2013)

DOI: 10.1103/RevModPhys.85.1083

\begin{abstract}
Microwave photons trapped in a superconducting cavity constitute an ideal system to realize some of the thought experiments imagined by the founding fathers of quantum physics. The interaction of these trapped photons with Rydberg atoms crossing the cavity illustrates fundamental aspects of measurement theory. The experiments performed with this "photon box" at Ecole Normale Supérieure (ENS) belong to the domain of quantum optics called "cavity quantum electrodynamics." We have realized the nondestructive counting of photons, the recording of field quantum jumps, the preparation and reconstruction of "Schrödinger cat" states of radiation and the study of their decoherence, which provides a striking illustration of the transition from the quantum to the classical world. These experiments have also led to the demonstration of basic steps in quantum information processing, including the deterministic entanglement of atoms and the realization of quantum gates using atoms and photons as quantum bits. This lecture starts by an introduction stressing the connection between the ENS photon box and the ion-trap experiments of David Wineland, whose accompanying lecture recalls his own contribution to the field of single particle control. I give then a personal account of the early days of cavity quantum electrodynamics before describing the main experiments performed at ENS during the last 20 years and concluding by a discussion comparing our work to other researches dealing with the control of single quantum particles.
\end{abstract}

\section{CONTENTS}

I. Control of Single Particles in a Quantum World

II. A Personal Account of the Early Days of Cavity Quantum Electrodynamics

III. Counting Photons Without Destroying Them: A New

Photon Box and Circular Rydberg Atoms

IV. Quantum Feedback

V. Field State Reconstruction

VI. SchrÖdinger Cat States of Light and Decoherence

Studies

VII. Quantum Information in Cavity QED

VIII. Perspectives for the ENS Work

IX. Relation to Other Domains in Quantum Information

Science

Acknowledgments

References

\section{CONTROL OF SINGLE PARTICLES IN A QUANTUM WORLD}

Quantum theory told us almost a hundred years ago that matter as well as light has a particle and a wavelike character. Atoms and subatomic particles, usually described as discrete entities, can behave as waves while light, generally described as an electromagnetic wave, is also made of discrete quanta,

*The 2012 Nobel Prize for Physics was shared by Serge Haroche and David J. Wineland. These papers are the text of the address given in conjunction with the award. the photons. This wave-particle duality leads to a strange quantum world in which atoms and light fields can exist in state superpositions, so to speak suspended between different classical realities. This situation is impossible to comprehend intuitively by our classical minds which have evolved to understand the macroscopic world surrounding us.

The fathers of quantum physics have been led to this strange description of the world by deductive reasoning based on the discovery of properties of matter and radiation which could not be explained by classical physics. The direct observation of the quantum strangeness, though, has been for a long time elusive. Bohr, Einstein, and Schrödinger described thought experiments in which they imagined that they manipulated and observed single quantum particles in order to reveal the counterintuitive behavior of nature at the microscopic level. Figure 1 shows for instance the famous photon box supposed to keep photons for a while and to release them on demand, imagined by Einstein and Bohr to illustrate their discussions (Bohr, 1949). This virtual experimental setup had many parts, among which was a clock to time a shutter which releases the photon, which was drawn on Bohr's instructions with exquisite realism.

While imagining this and other such experiments, the fathers of the theory could not envision that they would be one day feasible. From the 1930s, for sure, single particles could be detected and studied, for instance in bubble or cloud chambers or in accelerator experiments, but they were then observed by their traces or by the debris they produced after being smashed against each other in fiery collisions. In these experiments, their existence and properties were deduced so to 


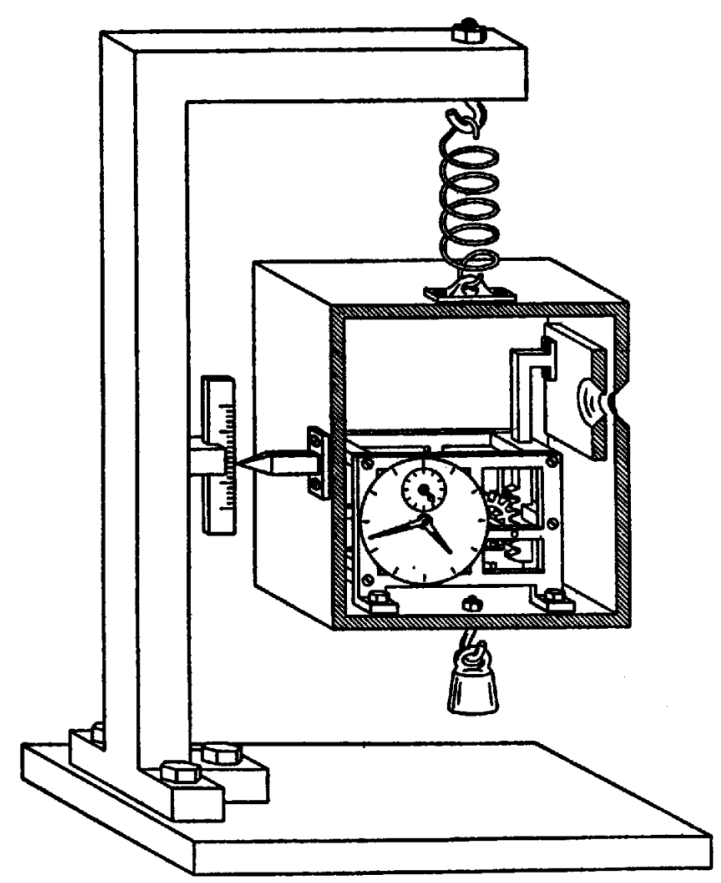

FIG. 1. The Einstein-Bohr photon box. From the Niels Bohr Archive.

speak postmortem. As Schrödinger wrote in 1952 about these studies: "It is fair to state that we are not experimenting with single particles, any more than we can raise Ichthyosauria in the zoo. We are scrutinising records of events long after they have happened" (Schrödinger, 1952).

In order to observe the quantum strangeness in the zoo, one had to manipulate particles of matter or light in a much more gentle way, without destroying them by the mere act of observation. Methods to achieve this subtle manipulation had to wait the development of tunable narrow band lasers, of fast computers, and of superconducting materials, which are all, in one way or the other, technologies emergent from quantum theory. Using these methods and working in the field of quantum optics, many groups in the world are now able to detect and manipulate single particles, while preservingeven exploiting - their quantum properties. The studies carried on by David Wineland and his team at the National Institute of Standards and Technology (NIST) in Boulder and by my group at the Laboratoire Kastler Brossel of Ecole Normale Supérieure (ENS) in Paris belong to this very active field of research.

In fact, the Boulder and Paris experiments represent the two sides of the same coin: the NIST group is trapping single charged atoms, called ions, in a configuration of fields produced by electrodes and uses laser beams to manipulate and detect the behavior of these particles of matter. In the ENS team we do the opposite, trapping photons in a cavity made of highly reflecting mirrors and using beams of atoms to manipulate, detect these particles of light, and study their evolution. The principle of these complementary experiments is sketched in Figs. 2(a) and 2(b). In both cases, we perform in vivo studies, trying to preserve the quantum properties of the manipulated systems.

A simple theoretical model, introduced by Jaynes and Cummings (Jaynes and Cummings, 1963) in the early days

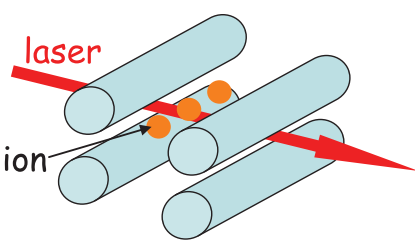

(a)

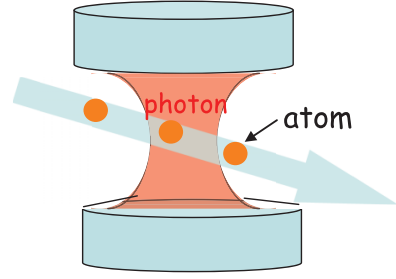

(b)
FIG. 2 (color). Scheme of the (a) NIST ion trap and the (b) ENS photon box.

of laser physics applies to both situations and contributes to unify these two lines of research. It describes the atoms as two-level spinlike systems interacting with a quantum oscillator. In the Boulder experiments, the ions' internal degrees of freedom (making up the "spin") are coupled to the external motion of the particles in the trap undergoing a mechanical oscillation, whose quanta are phonons. The coupling is achieved by irradiating the ions with properly tuned laser beams producing transitions between the ion internal energy levels accompanied by the absorption or emission of phonons. In the Paris work, the two-level atoms interact directly with a microwave field mode in the cavity, again a quantum oscillator whose quanta are photons. The coupling simply describes in this case the processes of photon absorption and emission by atoms crossing the cavity. A simple description of the Jaynes-Cummings model and its application to the ENS and NIST experiments can be found in the book Exploring the Quantum: Atoms, Cavities and Photons (Haroche and Raimond, 2006).

Figures 3(a) and 3(b) illustrate the closeness of the NIST and ENS approaches. They show experimental signals published in two papers which appeared back to back in 1996, in the same issue of Physical Review Letters. We had worked independently in Paris and Boulder on quite different setups and yet the recordings appear very similar. Figure 3(a) exhibits the "signature" of an ion oscillating in a state superposition of different vibration quanta in the Boulder trap (Meekhof et al., 1996) and Fig. 3(b) shows the corresponding signature of atoms having resonantly interacted with a small oscillating electromagnetic field containing a superposition of different photon numbers in the Paris cavity (Brune, Schmidt-Kaler et al., 1996). These signals, which represent transition probabilities between two ionic or atomic states versus time, are called "Rabi oscillations." Their shapes, exhibiting beating between sinusoidal oscillations corresponding to different numbers of quanta, reveal the graininess of the mechanical or field oscillator to which the atoms or the ions are coupled. As will be recalled below, exploiting such Rabi oscillations plays an important role in the manipulation of atomic and field states for quantum information processing.

\section{A PERSONAL ACCOUNT OF THE EARLY DAYS OF CAVITY QUANTUM ELECTRODYNAMICS}

The adventure of trapping and controlling single particles had in fact begun much before these Rabi oscillation experiments. David Wineland reminisces in his Nobel Lecture 


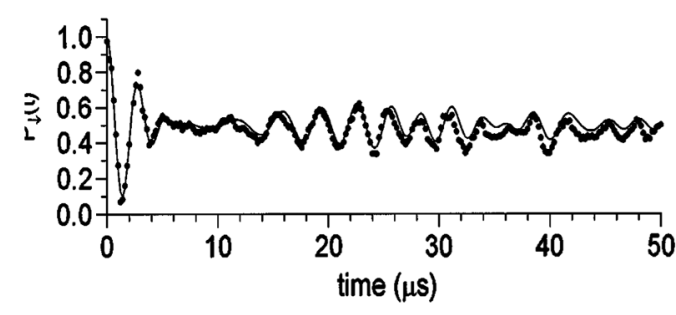

(a)

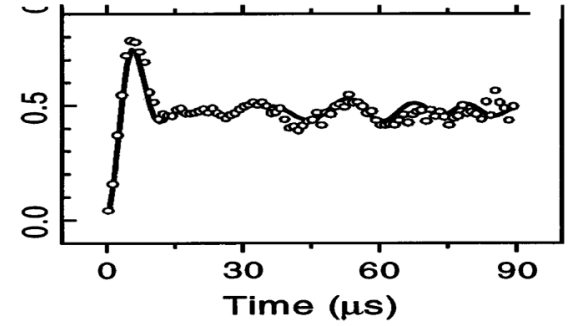

(b)

FIG. 3. Rabi oscillations in the (a) NIST and the (b) ENS experiments. From Brune, Schmidt-Kaler et al., 1996 and Meekhof et al., 1996.

about the early history of ion-trap physics. Here, I recall how my "photon box" experiments started, at a time when the similarity between these two lines of emerging research was not yet obvious.

I must first recall my thesis work (Haroche and CohenTannoudji, 1969; Haroche, 1971a, 1971b) under the supervision of Claude Cohen-Tannoudji in the Laboratoire de Spectroscopie Hertzienne de l'Ecole Normale Supérieure which became later the Laboratoire Kastler Brossel. It is indeed during this formative period that I learned that matter could be manipulated with light. I got acquainted with the optical pumping method invented by Kastler and Brossel which uses light beams to orient the magnetic moments of atoms and to detect their dance in radio-frequency fields. At that time, we were manipulating and observing large ensembles made of billions of atoms contained in a resonance cell. I was marveling at the fact that all our experiments were explained by the laws of quantum mechanics which had just been exposed to me in the illuminating lectures of my thesis advisor. We only had to trust that there were atoms in our cell, obeying collectively to these laws, even if we could not observe them individually.

At the same time, Claude and I developed the dressed atom formalism which explains the behavior of atoms exposed to radio-frequency fields described in terms of photons (CohenTannoudji and Haroche, 1969; Haroche, 1971a, 1971b). Quantizing the field in this context was a bold move since our fields contained huge numbers of light quanta and could well be described as classical continuous waves. The quantum picture, envisioning our atoms as being surrounded or "dressed" by clouds of photons, was directly inspired by the theory of quantum electrodynamics, which was alien to most atomic physicists. This approach turned out however to be very powerful and allowed us to discover many interesting phenomena in magnetic resonance which were not as clearly apparent in the classical approach (CohenTannoudji et al., 1970).

It was around that time that the dream of performing experiments involving small numbers of photons started to take shape in my mind. Thinking about a question of Anatole Abragam, who was a member of my thesis defense committee, I wondered whether it would be possible to design an experiment in which the dressed atom formalism would not be merely a convenient description of the physics, but a necessary approach to explain truly quantum phenomena. I had no idea of how to achieve this when I left Paris in 1972 for Stanford and my postdoctoral visit with Arthur Schawlow.

During this very exciting time, I witnessed the development of the first commercial tunable dye lasers, which were going to revolutionize atomic and molecular spectroscopy. These lasers, produced by Californian startup companies, were delivered as prototypes to neighboring universities, among which Stanford was probably served first. Having worked for my thesis research with classical lamps, I had to familiarize myself with these new sources of light. Working with Jeffrey Paisner, a graduate student of Art Schawlow, I used a pulsed dye laser to excite cesium atoms in a superposition of excited states and I observed the quantum beats in the fluorescence light subsequently radiated by the excited atoms (Haroche, Paisner, and Schawlow, 1973). These quantum beat studies drew again my attention to the important concept of state superposition which I had already encountered in a different context during my Ph.D. thesis work when studying resonances related to level crossings in the dressed atom energy diagram (Cohen-Tannoudji and Haroche, 1965).

Although the modulations of the fluorescence light characterizing quantum beats were observed on large ensembles of atoms, the basic phenomenon was a single atom interference effect. During the pulsed excitation and subsequent photon emission process, each atom was following several histories at once, leaving the atomic ground state and coming back to it while being transiently brought into different excited states. It was the indistinguishable character of these different paths which led to the final observation of the beats. Even if I was not able to do the experiment at that time, I knew the beats would still be there if I operated on a single atom, in the same way that a Young double slit experiment still works when particles cross the interferometer one at a time. Here again, I was intrigued by the challenge to observe quantum effects at the single particle level.

I imported to Paris in 1973 the tunable pulsed dye laser technology when I went back to ENS to take a permanent position at the National Center of Scientific Research (CNRS), before being appointed as a professor at Paris VI University in 1975. After my first quantum beat studies which were performed on states with small principal quantum numbers, it was natural to try to explore more excited atomic levels and I started to record quantum beats emitted by these levels in sodium (Haroche, Gross, and Silverman, 1974). Close to the atomic ionization limit, there was a very large number of levels forming a spectroscopic terra incognita of so-called Rydberg states with huge electronic orbits. The lasers offered us the opportunity to prepare and study these states and I was, as Daniel Kleppner at MIT and many other colleagues in Europe and in the USA, fascinated by the promising properties of these giant atoms, about which I give a few more details below. I became, in particular, interested by their extreme sensitivity to microwave radiation. 
I started performing microwave spectroscopy experiments on these atoms (Fabre, Goy, and Haroche, 1977, 1978), working with a small group of dedicated and bright graduate students. Michel Gross and Claude Fabre, who embarked later on their own independent and successful carriers, were followed shortly by Jean-Michel Raimond who stayed with me and accompanied me since then on this long research adventure. We were also lucky to get Philippe Goy, a condensed matter physicist wizard in millimeter wave technology, interested in our Rydberg atom-microwave spectroscopy experiments. The millimeter wave sources and analyzers that he developed for our research has led him to start a small company which sells to this day custom-made millimeter wave devices to laboratories throughout the world.

We were using for our experiments the simple setup sketched in Fig. 4: atoms flying in an atomic beam are excited into Rydberg states by lasers and, in the same region of space, they are irradiated by a microwave field. This field is confined in an open cavity made of copper mirrors facing each other, so as to define precisely the zone in which the atoms are irradiated. After having left the cavity, the atoms are ionized between two condenser plates in a ramp of raising electric field which reaches at different times the ionization threshold for the two energy states $e$ and $g$ connected by the microwave transition. In this way, the different ionization signals allow us to discriminate the two states. This very efficient and selective detection method, first introduced in Rydberg atom physics by Daniel Kleppner and his students (Dukas et al., 1975), is the one we still use today.

I realized in 1979 that if the atoms were initially in the upper state of the transition and the cavity tuned into exact resonance, it was not necessary to inject microwaves in order to observe a fast transfer between the two states. I immediately recognized that the ensemble of excited Rydberg atoms was spontaneously emitting in the cavity, realizing a pulsed maser (Gross et al., 1979). The surprising and striking feature was the very low threshold of this maser action, which required only a few hundred atoms as opposed to the billions of atoms involved in ordinary maser or lasers. This unusual order of magnitude was obviously due to the very strong coupling of Rydberg atoms to microwaves. In the conclusion of the paper reporting the result, we noted that this experiment opened the way to the study of even much smaller samples, provided we could use better cavities with higher $Q$ factor and longer field damping time.

In fact, we had just started our journey toward single atom and photon detection and manipulation. The field in which we had embarked, dealing with atoms and photons interacting in a space confined by mirrors, was called "cavity quantum electrodynamics" (cavity QED for short), a name coined by Daniel Kleppner around 1980. I immediately liked this denomination because it connected once more atomic physics to quantum electrodynamics, in the spirit of what Claude Cohen-Tannoudji and I had already attempted ten years earlier with the introduction of the "dressed atom" formalism. Experiments remained to be done to demonstrate that the term "quantum" was legitimate, but now at least we knew in which direction we had to go. The cavity containing the field became the critical ingredient to be improved and the Rydberg atoms the working horses allowing us to explore the quantum properties of these fields.

We performed several precise quantitative studies of pulsed Rydberg masers, focusing, in particular, on the evolution of the atomic ensemble during the emission process. Our experiment geometry, with all the atoms being symmetrically coupled to the same field, realized an ideal superradiant sample, a system that $\mathrm{R}$. Dicke had theoretically studied back in the 1950s (Dicke, 1954): the atoms initially in a fully excited state symmetric by exchange of atoms remained in such a state throughout the emission process, ending in the final state where all the atoms were in the lower state of the transition. This symmetry entailed a strong collective coupling of the atoms to the field and an emission time much shorter than the spontaneous emission time of a single atom. Our experiment allowed us to measure this shortened emission time and to study the whole dynamics of the emission process. By accumulating statistics over many realizations of an experiment performed with $N$ initially excited atoms, we recorded the probability versus time that $n$ of these atoms had been deexcited and thus $n$ photons emitted (see Fig. 5). This realized a first quantitative experimental demonstration of Dicke superradiance (Gross and Haroche, 1982; Raimond et al., 1982a).

We also showed that if the number of decaying atoms was increased beyond a certain limit, the emission started to exhibit a ringing feature, the atomic ensemble getting partially reexcited and undergoing damped oscillations while exchanging its energy with the field in the cavity (Kaluzny et al., 1983). This ringing regime of superradiance is described by an equation similar to that of a damped pendulum (Bonifacio, Schwendimann, and Haake, 1971). In another series of experiments, we studied also the opposite effect, namely, the collective absorption of blackbody radiation by Rydberg atoms initially prepared in the lower state of the
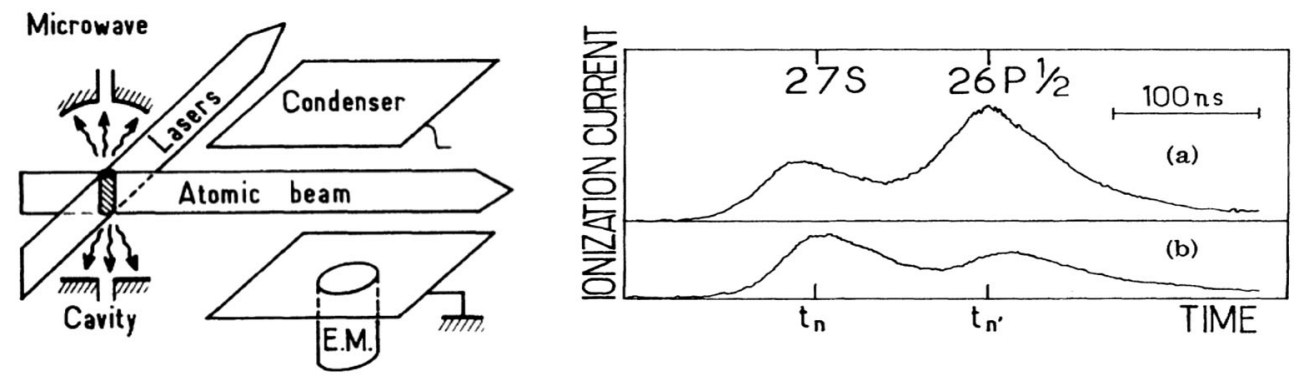

FIG. 4. The 1979 Rydberg atom maser experiment: Left: Sketch of the setup. Right: Time resolved atomic ionization signals discriminating the upper $(27 S)$ and lower $(26 P)$ states of the atomic transition. The bottom and top traces correspond to off-resonance and on-resonance cavity, respectively. From Gross et al., 1979. 


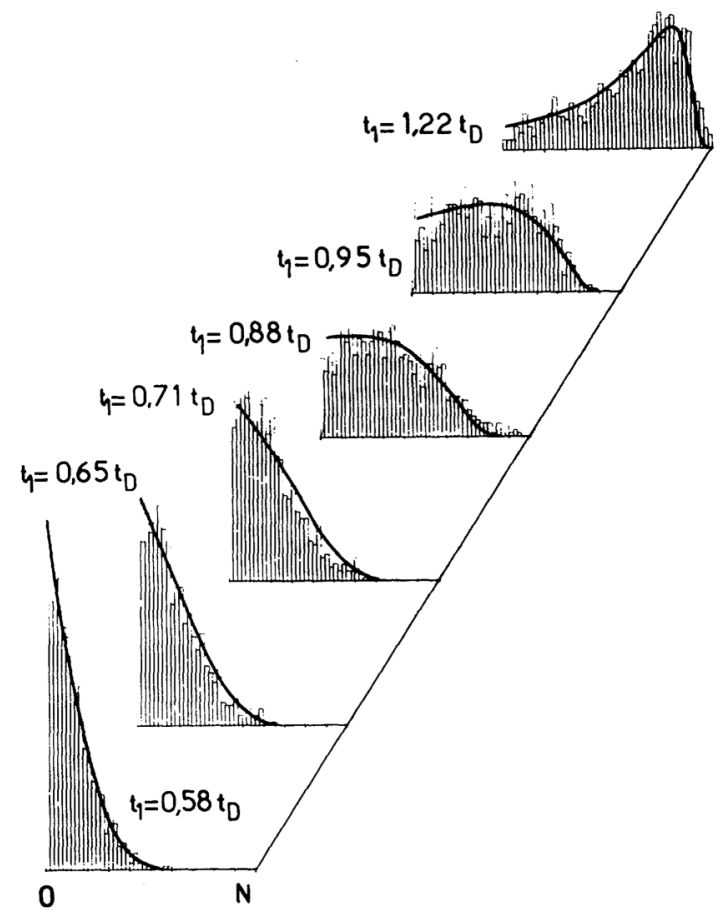

FIG. 5. Experimental demonstration of Dicke superradiance: histograms showing the probability that $n$ photons have been emitted by a sample of $N \sim 3200$ initially excited atoms at times increasing from bottom to top. Times are expressed in units of $t_{D}$, the average emission delay. The curves are theoretical. From Raimond et al., 1982a.

atomic transition resonant with the cavity field. In this process, the atoms behaved collectively as an ensemble of bosons remaining in a state symmetrical under particle exchange, leading to a heat capacity for our atomic system, much smaller than that of a classical gas (Raimond et al., 1982b). Incidentally, the symmetric states along which the atomic ensemble was evolving in these experiments were highly entangled, i.e., nondescribable as a product of single atom wave functions. We were well aware of this feature, even if the concept of entanglement and all its implications were not of central interest at that time.

We did not lose during these studies our primary goal which was to decrease the threshold of our masers to a single atom. In order to increase the reflectivity of our mirrors, we replaced the copper by superconducting niobium, cooled to a few Kelvin. By strongly reducing in this way the mirror absorption losses, we were able in 1983 to reach the situation where a single atom had its spontaneous emission rate enhanced by the presence of the cavity (Fig. 6). This effect predicted in 1946 by E. Purcell (Purcell, 1946), corresponded, as we noted, to the operation of a single atom transient maser (Goy et al., 1983). At about the same time, Daniel Kleppner and his students realized a complementary experiment proposed by Kleppner a few years earlier (Kleppner, 1981). They showed that the spontaneous emission of Rydberg atoms was inhibited, leading to a lengthening of the excited Rydberg state lifetime, when the atoms were confined between conducting plates excluding the radiation modes resonant with the atomic transition (Hulet, Hilfer, and Kleppner, 1985). Another demonstration of spontaneous emission inhibition had been performed a few months earlier by G. Gabrielse and

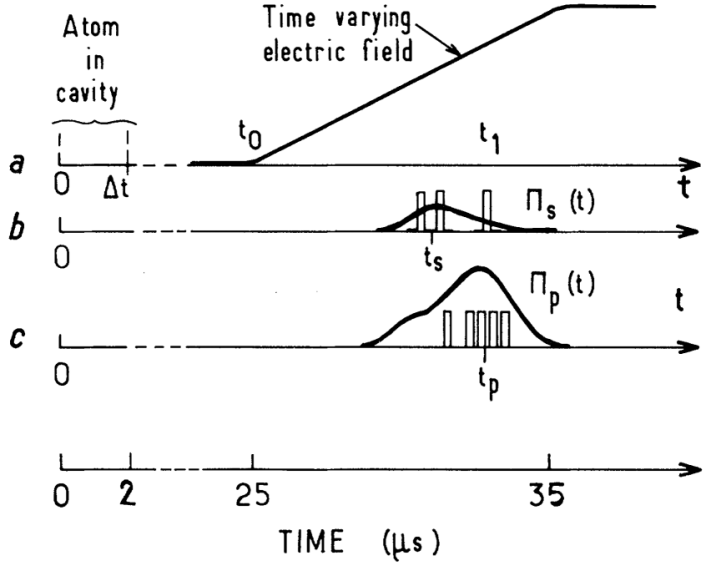

FIG. 6. Enhancement of spontaneous emission in a cavity: sketch illustrating the experimental time sequence: (a) ionization field ramp, (b) detection of the $23 S$ initial level, and (c) detection of the $22 P$ final level. From Goy et al., 1983.

H. Dehmelt who had shown that the decay of the cyclotron radiation of a single trapped electron was slowed down when it was located in the microwave cavity formed by the electrodes of the trap which was confining it (Gabrielse and Dehmelt, 1985).

A feature of our spontaneous emission enhancement experiment was intriguing me. What would happen if the cavity $Q$ factor was so large as to keep the emitted photon long enough for it to be absorbed by the atoms? As I noted in the lecture notes of a course I gave in Les Houches in 1982 (Haroche, 1984), this should lead to a regime of reversible exchange of energy between matter and radiation at the single particle level. If the atom was initially (at time $t=0$ ) in level $e$ and the cavity in vacuum, we expected, according to the Jaynes-Cummings model, that the atom + field system would evolve at time $t$ into the state $\cos \left(\Omega_{0} t / 2\right)|e, 0\rangle+$ $\left.\sin \left(\Omega_{0} t / 2\right) \| g, 1\right\rangle, \Omega_{0}$ being the so-called vacuum Rabi frequency, and the two symbols in each ket representing the atom's state and the number of photons in the cavity, respectively. If the cavity contained $n$ photons, a similar oscillation phenomenon was expected to occur between the states $|e, n\rangle$ and $|g, n+1\rangle$, at the larger frequency $\Omega_{n}=\Omega_{0} \sqrt{(n+1)}$. If the cavity field was in a superposition of photon number states, we expected this Rabi oscillation to exhibit a beating between components evolving at the incommensurate $\Omega_{n}$ frequencies, a phenomenon predicted theoretically by Eberly et al. (Eberly, Narozhny, and Sanchez-Mondragon, 1980) that we were to observe only in 1996, as recalled above. Our Holy Grail became in the early 1980s to reach this situation, now called the strong coupling regime of cavity QED.

We were however at a loss to improve the quality of our mirrors. The superconducting material was fine, but the mechanical properties of niobium made it difficult to get surfaces smooth enough to avoid photon scattering off small imperfections. While we were struggling with this technical problem, we got in 1984 news from Munich that a German colleague, Herbert Walther, working with his graduate student Dieter Meschede and G. Muller, a colleague expert in superconductivity, had reached our Holy Grail! They had had the bright idea to replace the open mirror structure by a closed 
cylinder, making it impervious to small irregularities in the inner walls and the lifetime of the photons was increased by several orders of magnitude.

Sending Rydberg atoms one at a time through this box, they realized a maser in which the field was building up into a steady state from the successive actions of single atoms crossing the apparatus one by one (Meschede, Walther, and Muller, 1985) (Fig. 7). The system clearly operated in the strong coupling regime. Walther called it a "micromaser." Numerous studies of this device were made during the 1980s and the 1990s, both on the theoretical and on the experimental side (Meystre, Rempe, and Walther, 1988; Rempe, SchmidtKaler, and Walther, 1990; Scully, Englert, and Walther, 1991). Herbert Walther, who sadly died in 2006, was a great leader in quantum optics who made with the micromaser an essential contribution to cavity QED. Dieter Meschede became my postdoc when I was working for a while part time at Yale University during the $1980 \mathrm{~s}$. He is now a physics professor in Bonn, Germany, doing beautiful experiments in cold atom physics and quantum information. Our ENS and Bonn groups have been exchanging ideas and sharing many students and postdocs along the years.

During our common time at Yale, Dieter and I performed in collaboration with Edward Hinds, Luigi Moi, and our students an experiment demonstrating the inhibition of spontaneous emission of an atom propagating between two mirrors separated by less than half the wavelength of the emitting transition (Jhe et al., 1987). This experiment, performed in 1986, was part of the thesis work of Won Jhe, a bright Korean student who has since become a professor in Seoul. It was a transposition to the optical domain of Kleppner's earlier experiment made in the microwave domain with Rydberg atoms (Hulet, Hilfer, and Kleppner, 1985). During the same period, my ENS group in Paris contributed to the micromaser studies by realizing, in 1987, a two-photon Rydberg atom maser operating with a closed cylindrical cavity, in which the light quanta were emitted by pairs (Brune et al., 1987) [a related two-photon laser had been realized a few years earlier (Nikolaus, Zangh, and Toscheck, 1981)]. The two-photon Rydberg maser was the Ph.D. subject of Michel Brune, an outstanding student whom we were lucky to keep in our group as a colleague since then.

At the time of these experiments, witnessing the successes of the Munich group, we were worried that our open cavity

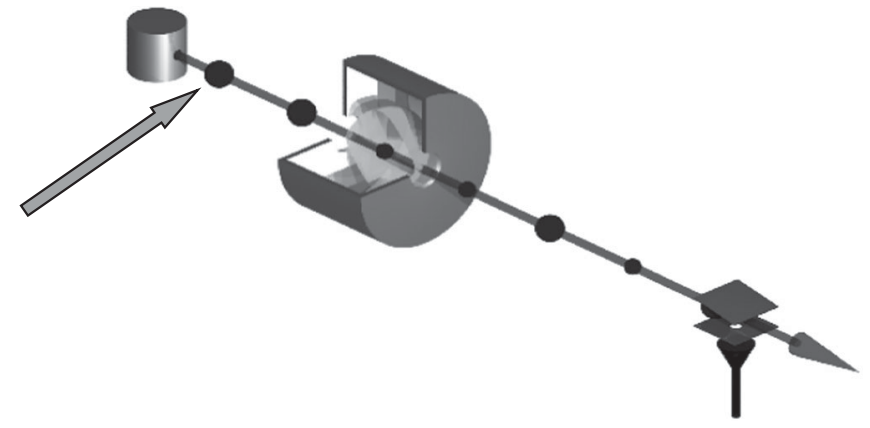

FIG. 7. Sketch of the Munich micromaser: atoms excited by a laser into a Rydberg state (arrow at left) cross one by one a cylindrical superconducting cavity, before being detected by field ionization (at right). mirrors were leading us into a dead end. We were reluctant though to adopt definitively the closed cavity solution, because it had a serious problem for the experiments which we were planning: atoms had to enter and exit through small holes, passing near metallic surfaces producing stray electric fields. This proximity with metals was strongly perturbing the very sensitive Rydberg atoms, especially when they were prepared in superposition of states. The phase of the superposition was strongly affected by the stray fields, making it impossible to manipulate easily the atomic and field quantum states. The alternative solution was to stick to open cavity structures, in which atoms avoided to pass close to metallic surfaces, but we had then to solve the surface smoothness issue. It took us about 20 years to be able to solve this problem progressively and to realize the photon control and manipulation experiments which have been recognized by the Nobel prize. In order to describe them, I will abandon the historical approach and adopt a more didactic point of view. A presentation of the early developments in cavity QED can be found in a Physics Today article that I coauthored with Daniel Kleppner in 1989 (Haroche and Kleppner, 1989).

\section{COUNTING PHOTONS WITHOUT DESTROYING THEM: A NEW PHOTON BOX AND CIRCULAR RYDBERG ATOMS}

Our ambition, back at the end of the 1980s, became to generate photons in a high- $Q$ cavity and to observe and manipulate these photons without destroying them. To achieve this feat, we had to overcome a difficulty: usually the detection of light belongs to the "postmortem" kind of procedure alluded to by Schrödinger in the quotation recalled in the first section of this Lecture. When you see light, the photons which strike your eye, your camera, or your photodetector are converted by the photoelectric effect into an electron, or some kind of electric current and the photon is destroyed in the process. The situation is not essentially different from the brute force collisions occurring in a cloud chamber or an accelerator of particles. Seeing light amounts to collecting the debris of the photons colliding with a photosensitive surface. If you detect a click into your photon counter, the photon is no longer there and replaced by the vacuum, zero photon instead of one!

What we were looking for instead was a process by which a click into our nondestructive detector would leave the photon intact afterward, allowing the measurement to be repeated again and again, on the same photon. The idea of such a quantum nondemolition (QND) measurement for the electromagnetic field energy had been proposed by V. Braginsky and colleagues in the 1970s (Braginsky et al., 1977; Braginsky and Vyatchanin, 1981; Braginsky and Khalili, 1996) In order to adapt the method to the counting of light quanta, a special kind of atomic detector was required, which was transparent to radiation, but still sensitive enough to register an imprint of a single photon. And we needed also a very good photon box, an open structure keeping the photons for a very long time, enabling us to repeat again and again the measurement with successive atoms crossing the box one by one. This brought us back to the Einstein-Bohr photon box, but in a radically new context. 
The box we came up with is not like the one drawn by Bohr 85 years ago, but, interestingly enough, it is indeed coupled to a clock, a very special clock in fact, as described below. The solution to the cavity defects which had plagued us in the 1980 s was found by successive improvements. We first succeeded to reach photon lifetimes in the several hundred microsecond to a millisecond range by surrounding the niobium mirrors with an aluminum ring which reflected the field scattered on mirror imperfections back into the cavity mode. But the real breakthrough came from a collaboration with scientists of the Center of Atomic Energy (CEA) in Saclay. They worked on superconducting cavities for particle accelerator experiments and were experts in the art of designing high- $Q$ microwave resonators, albeit at smaller frequencies than the ones (around $50 \mathrm{GHz}$ ) that we were using. With their help, we finally realized mirrors out of copper, which can be machined with high precision, reducing the surface roughness to a few nanometers and we sputtered a thin layer of superconducting niobium on top of them. By combining in this way a precise geometry with a high electric conductivity, we obtained mirrors with quasispherical surfaces, whose quality steadily improved as we refined our procedures, without any longer requiring an aluminum ring around the mirrors. We finally reached a lifetime of $130 \mathrm{~ms}$ in 2006 (Kuhr et al., 2007) with a fully open cavity structure (Fig. 8). Photons travel between these mirrors over about $40.000 \mathrm{~km}$ on a folded trajectory and survive long enough for thousand of atoms, crossing the cavity one by one, to interact with them.

In order to probe the photons, we send across the cavity a special kind of Rydberg atoms, called "circular," in which the outer electron orbits on a circle of large diameter, about 1000 times bigger than an ordinary ground state atom. These excited rubidium atoms are prepared with lasers and radio-frequency excitation, using a modified version (Nussenzveig et al., 1993) of a procedure invented by Daniel Kleppner and Randy Hulet at MIT in 1983

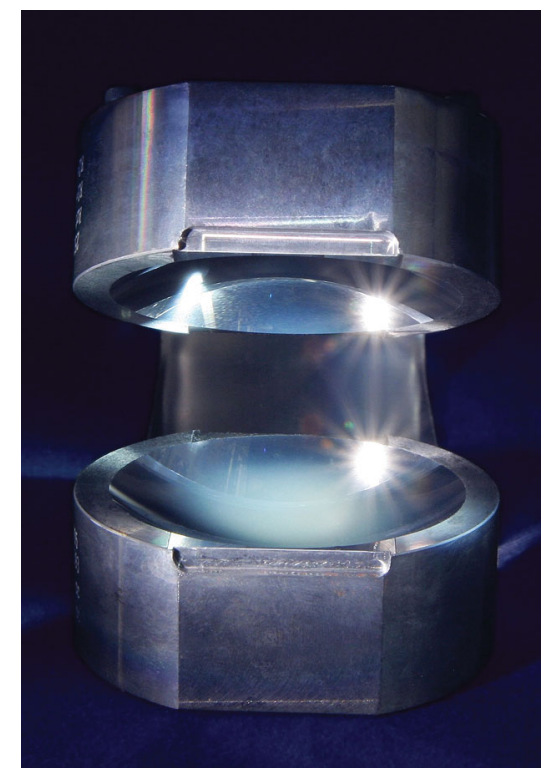

FIG. 8 (color). The ENS photon box (photograph by Michel Brune). The mirrors have a diameter of $5 \mathrm{~cm}$ and are $2.7 \mathrm{~cm}$ apart (for a clear view, they are more separated in this picture than in the actual experimental setup).
(Hulet and Kleppner, 1983). According to quantum theory, the orbiting Rydberg electron is also a wave, which has a de Broglie wavelength and the condition of a stable orbit is that there is an integer number of these wavelengths along the circumference. This number, called the principal quantum number of the Rydberg atom, is equal to 51 or 50 in our experiments (these Rydberg states are called, respectively, $e$ and $g$ in the following). The advantage of these circular Rydberg states of maximal angular momentum over the states of small angular momentum employed in our earlier experiments is their very long natural lifetime, of the order of $30 \mathrm{~ms}$ for the states with principal quantum number 50 or 51 . This lifetime, of the same order of magnitude as the photon lifetime in our cavity, allows us to neglect in first approximation the atomic decay processes during the interaction time between the atoms and the cavity field.

In the $e$ and $g$ Rydberg states, the circulating de Broglie wave has a uniform amplitude, resulting in an electron charge density centered at the atomic nucleus, yielding a zero electric atomic dipole [see Fig. 9(a)]. In order to prepare an electric dipole, a pulse of resonant microwave can be applied to the atom, bringing it in a superposition of the two adjacent $e$ and $g$ states, with, respectively, 51 and 50 nodes in their wave function. This superposition of states can be referred to as a "Schrödinger cat" because it implies an atom at the same time in two levels, reminding us of the famous cat that Schrödinger imagined suspended between life and death. A better name would be a "Schrödinger kitten," because it is made of a single atom and thus very small. The two de Broglie waves making up this "kitten" interfere constructively at one end of the orbit and destructively at the other end, resulting in a net electric dipole, rotating in the orbit plane at $51 \mathrm{GHz}$ [see Fig. 9(b)]. This dipole behaves as a rotating antenna extremely sensitive to microwave radiation. It can also be described as the rotating hand of a clock, ticking at $51 \mathrm{GHz}$.

When microwave radiation, nonresonant with the transition between the two states $e$ and $g$, impinges on the atom, it cannot absorb it and hence the photons remain intact, ensuring the nondemolition character essential to our experiments. However, the effect of this nonresonant light is to shift slightly the atomic energy levels and hence to alter the rotating frequency of the atomic dipole, our clock hand. This light shift effect had been discovered in 1961 by Claude Cohen-Tannoudji in his seminal optical pumping studies

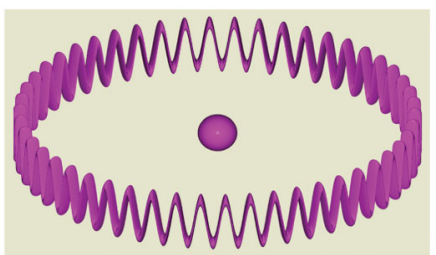

(a)

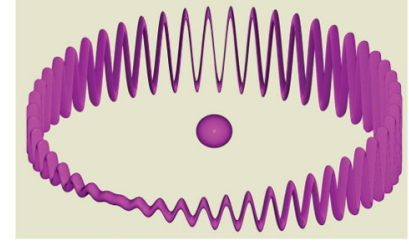

(b)
FIG. 9 (color). Circular Rydberg atom electron waves: (a) In an energy eigenstate, the de Broglie electron wave has a uniform amplitude around the circular orbit, resulting in a zero electric dipole; and (b) in a superposition of adjacent circular Rydberg states, the de Broglie waves interfere positively on one side of the orbit and destructively on the other side, yielding a net electric dipole which rotates in the orbit plane. 


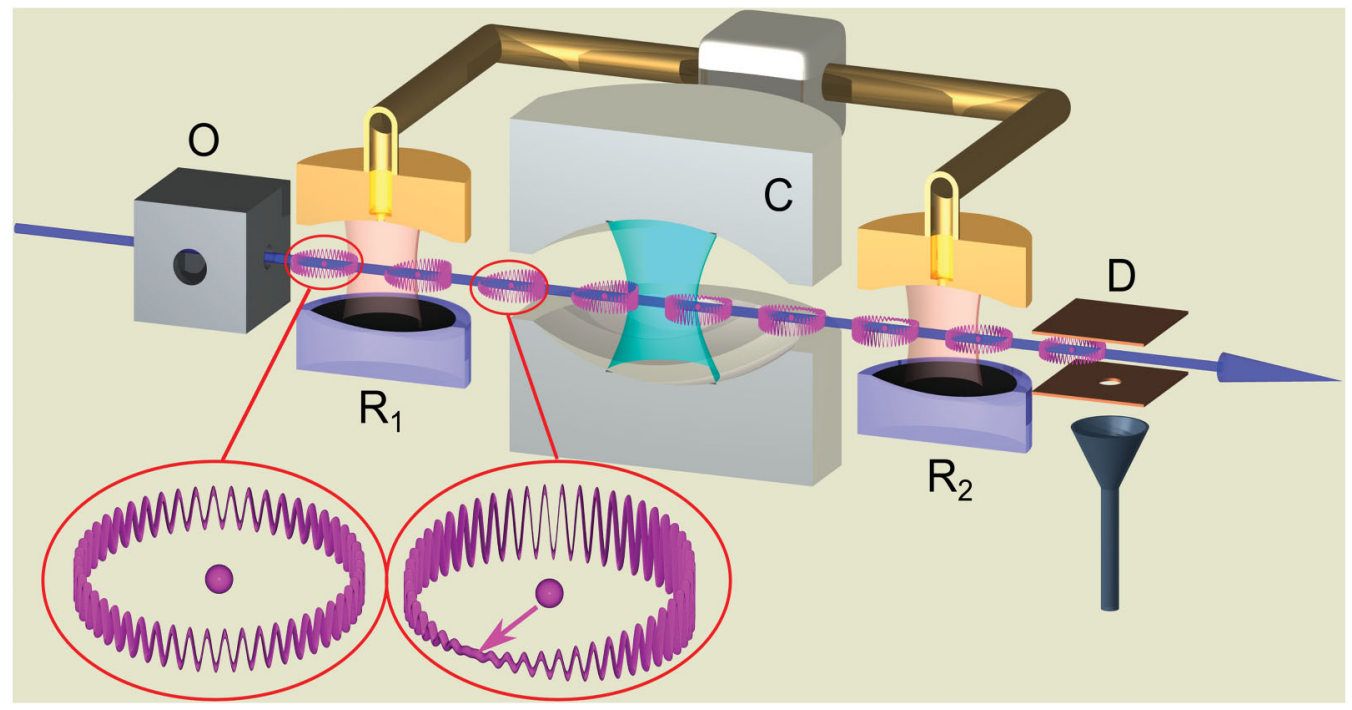

FIG. 10 (color). The cavity QED Ramsey interferometer setup. The insets show sketches of the circular atom in an energy eigenstate (left) and in a superposition state after interaction with the microwave pulse in $R_{1}$ (right). From Haroche, Brune, and Raimond, 2013.

(Cohen-Tannoudji, 1962a, 1962b). Light shifts are proportional to the field energy, i.e., to the photon number. Being inversely proportional to the atom-cavity field detuning, they can be maximized by tuning the cavity close enough to resonance (typically $100 \mathrm{kHz}$ away from the atomic transition frequency in our experiments) but far enough to avoid any photon absorption or emission process. In the case of Rydberg atoms, the effect per photon is then very large, resulting in a phase shift of the atomic dipole after the atom leaves the cavity which can reach the value of $180^{\circ}$, the dipole jumping in two opposite directions when the photon number changes by one unit. Measuring this phase shift amounts to counting the photon number without destroying the light quanta. Let us note that these light shifts play an essential role in other atomic physics and quantum optics experiments. They are at the heart of the methods used to trap and cool atoms in laser light, which were recognized by the Nobel Prize awarded to Claude Cohen-Tannoudji, William Phillips, and Steven Chu in 1997 (Chu, 1998; Cohen-Tannoudji, 1998; Phillips, 1998).

In order to measure these shifts, we followed a proposal that we made in 1990 (Brune et al., 1990). We built an atomic interferometer around our photon storing cavity (Fig. 10). The atoms, prepared in the circular state $e$ in the box $O$, cross the cavity $C$ one by one before being detected by field ionization in $D$. Essential to the experiment, two auxiliary microwave zones $R_{1}$ and $R_{2}$ are sandwiching the cavity $C$. In the first one, the atoms are prepared in the state superposition of $e$ and $g$, a
Schrödinger kitten state. This procedure amounts to starting a stopwatch, giving to the atomic dipole, i.e., to the clock hand, its initial direction. The atomic dipole then rotates as the atom crosses the cavity, until a second microwave flash, applied in $R_{2}$, is used to detect the direction of the atomic dipole at cavity exit, thus measuring the phase accumulation of the clock.

The combination of the two separated microwave resonators $R_{1}$ and $R_{2}$ is known as a Ramsey interferometer. The device had been invented in 1949 by Norman Ramsey (Ramsey, 1949) (who was to become later the Ph.D. advisor of Daniel Kleppner and David Wineland). The method of separated field pulses is now used in all atomic clocks working on a hyperfine microwave transition between two atomic levels. The excitation by the two successive pulses induces a sinusoidal variation of the transition probability when the microwave frequency is scanned around resonance. This socalled "Ramsey fringe" signal is used to lock the microwave frequency to the atomic transition. In our experiment, the Ramsey interferometer is counting photons by detecting the perturbing effect they produce on the fringes of a special atomic clock, made of microwave sensitive Rydberg atoms (Haroche, Brune, and Raimond, 2013). If the phase shift per photon is set to $180^{\circ}$, the Ramsey fringes are offset by half a period when the number of photons changes by one. The interferometer is set at a fringe maximum for finding the atom in $e$ if there is one photon in the cavity. The second pulse then transforms the state superposition of the atom exiting the

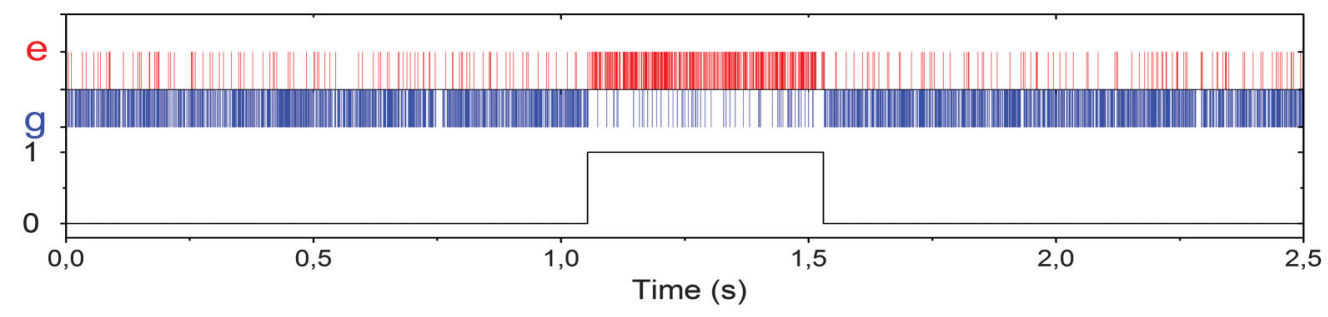

FIG. 11 (color). QND detection of a single photon: the sequence of single atom events (upper trace) detects the sudden change of the photon number (lower trace), revealing the birth, life, and death of a single light quantum. Photon lifetimes are random, with an average equal to the cavity field damping time of $130 \mathrm{~ms}$. The photon shown here had an exceptionally long lifetime of about 500 ms. From Gleyzes et al., 2007. 
(a)

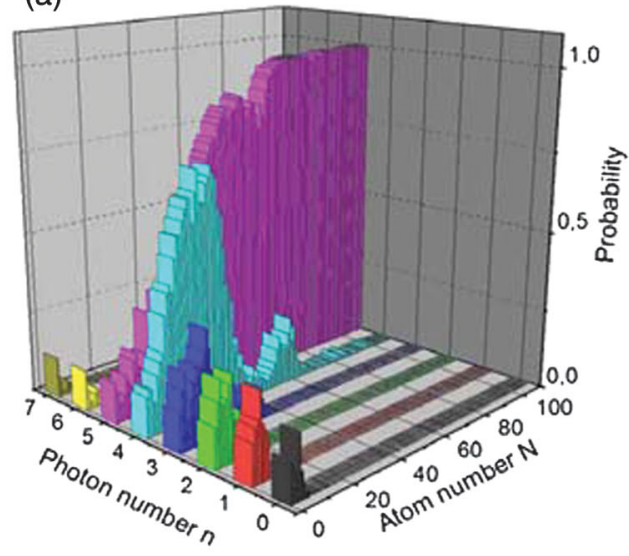

(b)

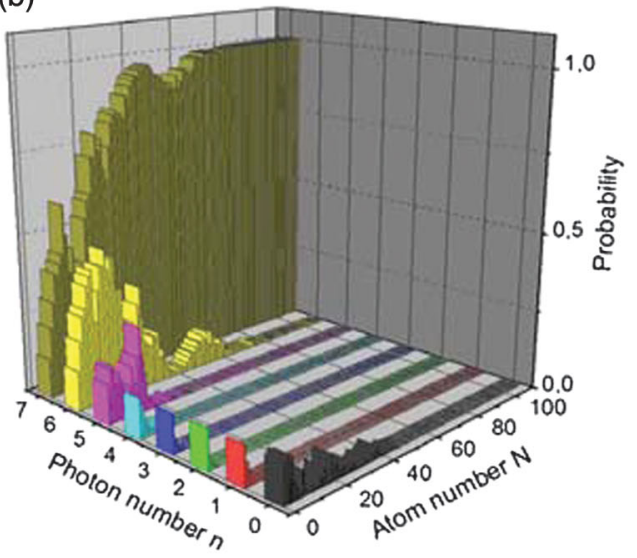

FIG. 12 (color). Progressive field state collapse induced by QND photon counting: (a) Evolution of the inferred photon number probability distribution as the number of detected atoms increases, in a realization of the experiment leading to the Fock state $n=5$; (b) evolution of the same distribution in a realization leading to $n=7$. From Guerlin et al., 2007.

cavity $C$ either in state $e$ (if there is one photon) or in state $g$ (if there is zero photon), this state being finally detected by the field ionization detector. The final atomic state $g$ or $e$ is thus correlated to the photon number, 0 or 1 .

Figure 11 shows the detection events ( $g$ and $e$, respectively, represented by blue and red bars) as atoms cross a cavity cooled at $0.8 \mathrm{~K}$, which, according to Planck's law, contains either the vacuum ( $95 \%$ of the time) or one photon (5\% of the time) (Gleyzes et al., 2007). One clearly sees when a photon pops inside the cavity, stays for a while, and then disappears. Because of noise and imperfections, the correlation between the photon number and the atomic signal is not perfect, but a simple majority test allows us to reconstruct without ambiguity the evolution of the photon number (lower trace in the figure). The sudden change of the photon number is a quantum jump, a phenomenon predicted long ago by quantum theory and observed in the 1980s in trapped ions, as described by David Wineland in his lecture. It is here observed for the first time for light quanta. The figure also shows that hundreds of atoms see the same photon between two quantum jumps, which demonstrates that our detection method is QND for the field. Note that these field oscillator jumps bear a strong similarity to the quantum jumps between the cyclotron oscillator states of a single electron, which were also monitored by a QND procedure (Peil and Gabrielse, 1999).

Our photon counting method can be extended to count larger numbers of light quanta (Guerlin et al., 2007). We start by injecting inside the cavity a small coherent field, superposition of photon number states comprised between 0 and 7 . This field is produced by scattering on the edges of the cavity mirrors a microwave pulse radiated by a classical source. This leads to the capture of a few photons which survive between the mirrors long after the source has been switched off. We then just need to send a sequence of atoms across the cavity, each carrying away a bit of information about the field. The phase shift per photon is optimally adjusted to a value such that different photon numbers correspond to well-separated atomic dipole directions at the cavity exit. At the start of the experiment, we have no idea about the photon number and we assume a flat probability distribution, as shown by the histogram on the left of Figs. 12(a) and 12(b), giving equal weights to the probability of having from 0 to 7 photons in the cavity. As successive atoms provide information, our knowledge about the field evolves until finally a single photon number is pinned down [the single peaked histograms on the right of Figs. 12(a) and 12(b) correspond to $n=5$ and 7 photons, respectively].

The evolution of the inferred probability distribution is obtained by a Baysian argument developed in detail by Haroche and Raimond (2006): each atom's measurement provides information about the atomic dipole direction and allows us to update our knowledge of the photon number distribution. This experiment shows, so to speak, in real time, the measurement-induced "wave function collapse" which appears here as a progressive process transforming a flat histogram into a single peak. The field, initially in a superposition of different photon number, is projected by the mere acquisition of information into a photon number state, a socalled Fock state of well-defined energy. The process is random, as shown by the two realizations of Figs. 12(a) and 12(b) which have produced different results. The statistics of a large number of measurements reconstructs the photon probability distribution of the initial state (Fig. 13). It obeys

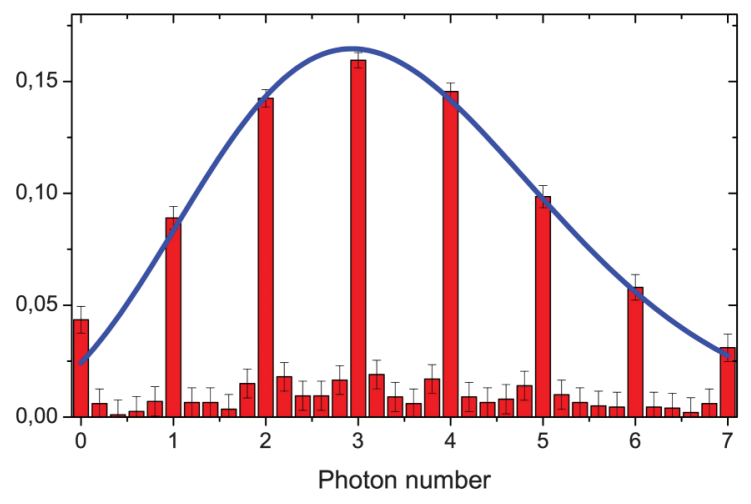

FIG. 13 (color). Photon number distribution of a coherent state with an average number of 3.4 photons reconstructed by statistical analysis of 3000 QND measurement sequences. From Guerlin et al., 2007. 
a
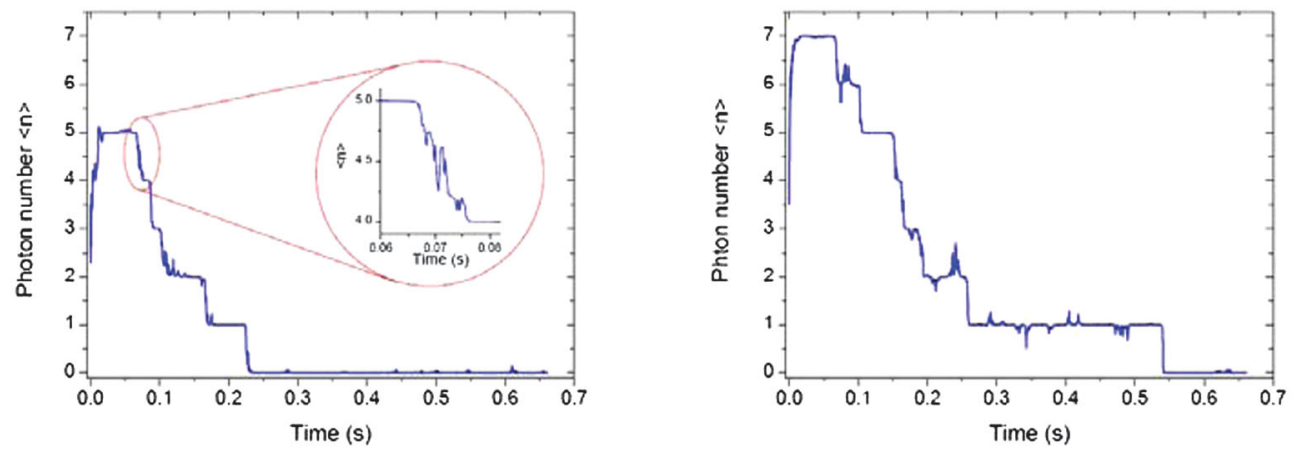

b
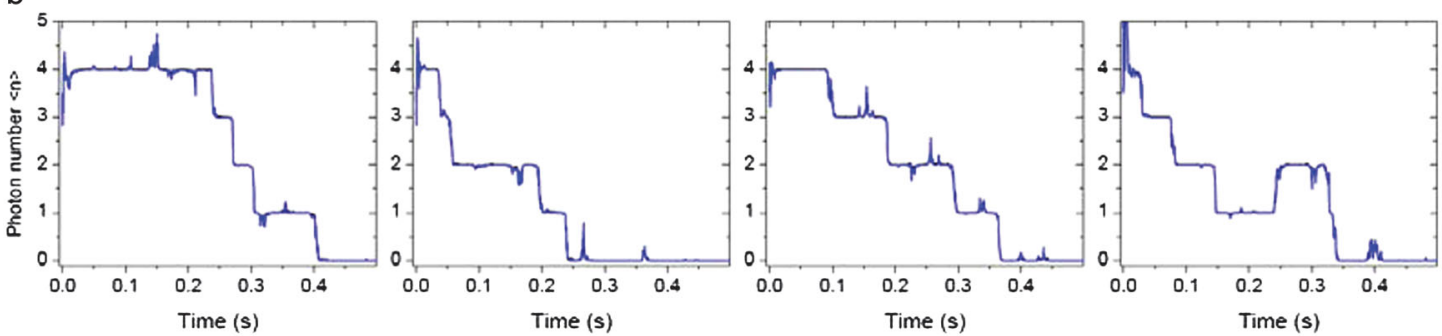

FIG. 14 (color). Field quantum jumps recorded by QND photon counting: (a) Evolution of the photon number following field collapse into $n=5$ (left) and $n=7$ (right). The zoom in the inset shows that, in order to record a quantum jump, the measurement requires the detection of several atoms, over a few millisecond time interval. (b) Four random photon number "trajectories" following field collapse into $n=4$. The different recordings illustrate the fluctuating nature of the field decay process. The trace at right exhibits an upward jump due to the transient appearance of a thermal photon in the cavity (cooled at $0.8 \mathrm{~K}$ ). From Guerlin et al., 2007.

a Poisson law, as expected for a coherent state produced by a classical source of radiation (Glauber, 1963).

Once a photon number has been pinned down, its ensuing evolution can be observed by continuing the measurement with subsequent atoms on the same realization of the experiment. We then observe the succession of quantum jumps leading the field inexorably back to vacuum, due to photon losses in the cavity walls (Guerlin et al., 2007). Figure 14 shows the trajectories followed by fields containing initially different numbers of photons. A statistical analysis of these trajectories has allowed us to measure the Fock state lifetimes. Fluctuating randomly from one preparation to the next, the lifetime of the $n$-photon number state is distributed according to an exponential probability law with the time constant $T_{c} / n$, where $T_{c}$ is the cavity field energy damping time (Brune et al., 2008). The $1 / n$ variation of this lifetime is a manifestation of the increasing fragility of these nonclassical states of radiation when their energy increases. They share this feature with Schrödinger cat states of light (see below).

\section{QUANTUM FEEDBACK}

The ability to measure a quantum system without destroying it opens the possibility to react back on it and to drive it deterministically toward a desired state. Soon after achieving our QND photon counting experiments, we proposed with Pierre Rouchon from the Ecole des Mines (Paris) and his students to implement this quantum feedback strategy to "tame" a quantum field, preparing and maintaining it in a predetermined Fock state for an indefinite time (Dotsenko et al., 2009). We then realized two versions of this experiment (Sayrin et al., 2011; Zhou et al., 2012). The Ramsey interferometer, acting as a QND photon counter, is used as a sensor providing information to a computer which estimates in real time the field state and decides how to react back on it in order to steer it toward the photon number "target" state. Once this state has been reached, the procedure monitors the quantum jumps and immediately corrects their effects by bringing back the system into the target state. The method is analogous to juggling. The juggler looks at the balls which he wants to keep on an ideal trajectory. His eyes are the sensors and the visual information, processed by his brain, is used to determine the correcting actions of his hands, the actuators. In the quantum version, the classical balls are replaced by photons bouncing between the mirrors. The quantum eye is the Ramsey interferometer, giving information on the state of the field to the computer "brain" which controls the experiment.

The quantum juggler faces a challenge, absent in the classical game. While looking at balls has no effect on them, the mere fact of observing the photons has an unavoidable backaction, as required by the projection postulate of quantum physics. This backaction is random, since its effect depends upon the unpredictable outcome of measurements. Once an atom has been detected though, the backaction on the field state is determined by projecting the field state according to the atomic measurement result. The computer can thus update the field state in real time, based on the results of successive measurements. At each step, it evaluates a distance between the actual state and the desired one, and it computes an adapted response to minimize this distance. As in classical feedback, the procedure is implemented in loops involving a succession of detection and corrections, until the target state is reached. It is then carried on to detect quantum jumps and correct for their effect.

What is the "hand" of this juggling game? In one version of the experiment (Sayrin et al., 2011), it is a microwave source used to periodically inject small microwave fields in $C$ 


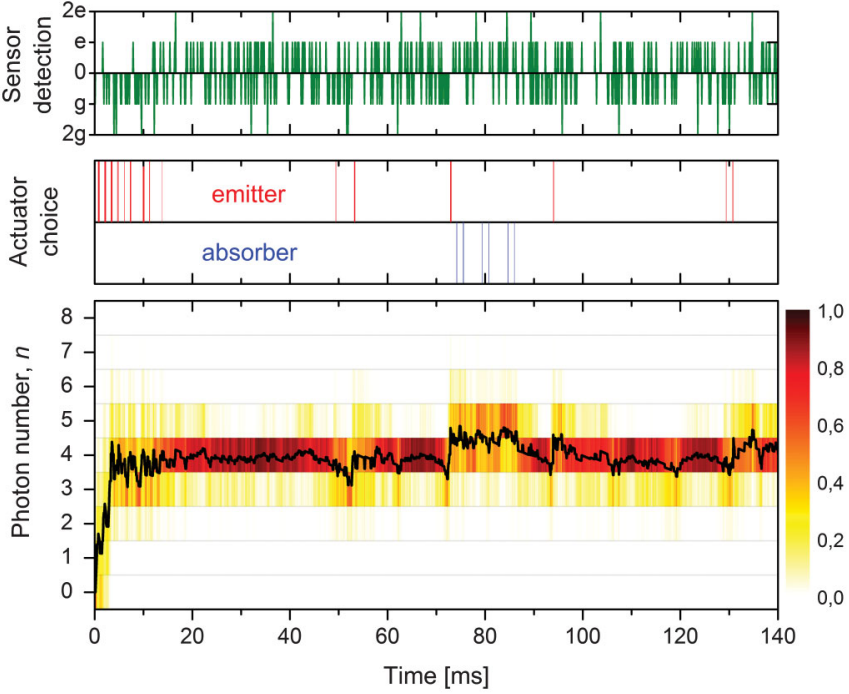

FIG. 15 (color). Stabilization by quantum feedback of the $n=4$ Fock state. The upper and middle frames show the sequences of sensor atoms and actuator atom detections (sensor, emitter, and absorber atoms indicated by green, red, and blue bars, respectively). The lower frame shows the resulting evolution of the photon number distribution as estimated by the computer driving the experiment (probabilities represented by the color code indicated at right). From Zhou et al., 2012.

which, depending upon their phase, increase or decrease the field amplitude. In another version (Zhou et al., 2012), the actuator is realized by atoms, tuned in exact resonance with $C$. They emit (if they enter $C$ in $e$ ) or absorb one photon (if they enter in $g$ ). The actuator atoms belong to the same atomic beam as the sensors. To make them resonant during the correcting stages of the feedback loop, the detuning between the atoms and the cavity field is momentarily canceled and the Ramsey pulses $R_{1}$ and $R_{2}$ deactivated. A feedback loop involves a sample of a few sensor atoms followed by a few actuators. Quantum feedback signals corresponding to the stabilization with atomic actuators of the $n=4$ Fock state are shown in Fig. 15. The cavity is initially in vacuum and the computer orders to send several emitter atoms to build the field, which stops growing when the target is reached. Photon jumps occurring at later times are corrected by emitter atoms. When the correction overshoots, absorber atoms are used. On average, the photon number is stabilized, with small residual fluctuations around the target $n$ value. These experiments open interesting perspectives for the deterministic preparation and protection against decoherence of other nonclassical states of radiation.

\section{FIELD STATE RECONSTRUCTION}

QND measurements, repeated on many realizations of the same field, have allowed us to reconstruct its photon number distribution $P(n)$, which, for a coherent field, is a Poisson function centered around its mean photon number value $\langle n\rangle$ (see Fig. 13). These $P(n)$ histograms provide only partial information about the field. Describing the light intensity and its fluctuations, they are insensitive to field coherences. In general, a field state is described by a density matrix $\rho$, whose diagonal elements $\rho_{n n}$ in the Fock state basis are the $P(n)$ probabilities, and the off-diagonal ones, $\rho_{n n^{\prime}}$, describe the field coherence. Expressed in terms of photon numbers, the $P(n)$ probabilities are "1D" objects while the $\rho_{n n^{\prime}}$ coherences are " $2 \mathrm{D}$ " entities. Reconstructing coherences from the measurement of photon number probabilities, i.e., "going from $1 \mathrm{D}$ to $2 \mathrm{D}$ " in the representation of the field, is analogous to going from $2 \mathrm{D}$ to $3 \mathrm{D}$ in photography.

The photon number distribution of a field state is indeed like a flat photo, obtained by recording the light intensity that the object has scattered into the lens of a camera. To add an extra dimension and achieve a full reconstruction, one must realize a hologram by adding phase information to the one provided by the intensity recording (Gabor, 1971). In photography, this is achieved by interfering the scattered light with a reference beam-a small fraction reflected off the main laser beam illuminating the object. The interference pattern recorded on the hologram is a Fourier transform of the object. When illuminated by a laser beam similar to the one which has produced it, the hologram reproduces the appearance of the object by inverse Fourier transformation.

Similarly, the full 2D $\rho_{n n^{\prime}}$ information contained in the quantum state of a field can be reconstructed by mixing this field with reference fields of various phases and amplitudes and by reconstructing the photon number distributions of these interfering fields. This procedure is called quantum tomography (Smithey, Beck, and Raymer, 1993). In our cavity QED experiments, the Rydberg atom Ramsey interferometer is used to perform these state reconstructions (Deléglise et al., 2008). Identical copies of the field are prepared, then admixed with reference coherent fields produced by a classical source. QND photon counting of the resulting "mixed fields" are then performed. From the data accumulated on many realizations with reference fields of different phases and amplitude, enough information is collected to reconstruct $\rho$.

To represent the field state, it is convenient to choose, instead of $\rho$, an alternative description. The field state is formally equivalent to the state of a mechanical oscillator evolving in a parabolic potential. Its state is represented by a real Wigner function (Schleich, 2005; Haroche and Raimond, 2006) taking its values in the oscillator phase space (the coordinates in this space being the position $x$ and momentum $p$ of the fictitious oscillator, corresponding to "field quadratures"). This function, which generalizes for the quantum field the classical concept of probability distribution in phase space, contains the same information as $\rho$, to which it is related by Fourier transformation. To keep the holographic analogy, the Wigner function is to the density matrix what the hologram is to the direct image of an object. Its interfering patterns directly reveal the main features of the quantum field.

Figure 16 shows the Wigner functions of two field states experimentally reconstructed by Ramsey interferometry (Deléglise et al., 2008). Figure 16(a) shows the Wigner function of a coherent state with $\langle n\rangle=2.5$ photons on average. It is a Gaussian peak centered at the point in phase space whose polar coordinates correspond to the amplitude and to the phase of the field. This positive function, which contains more information than the photon probability distribution in 
(a)

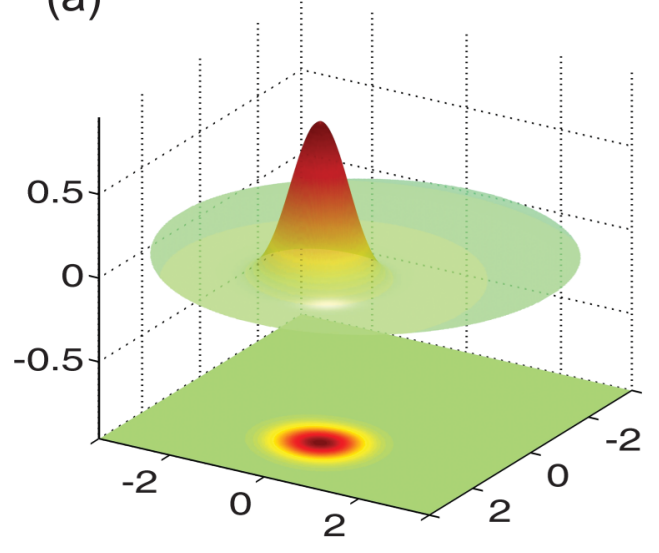

(b)

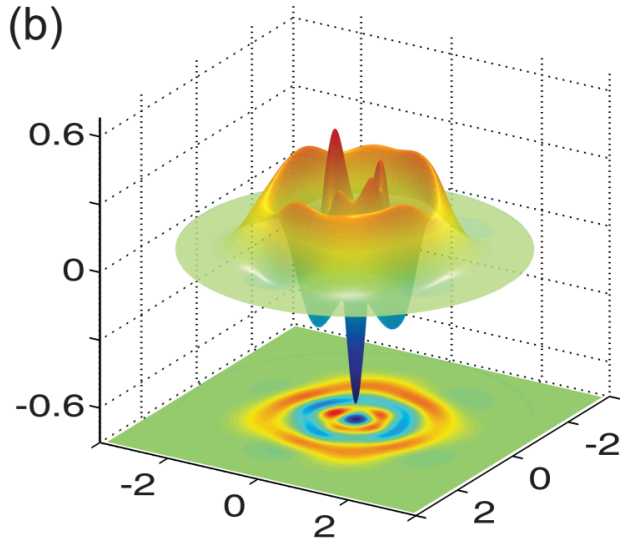

FIG. 16 (color). Experimental Wigner functions represented in 3D and 2D: (a) coherent state with $\langle n\rangle=2.5$; (b) $n=3$ Fock state. From Haroche, Brune, and Raimond, 2013.

Fig. 13, can be interpreted as a classical probability distribution in phase space, with uncertainties in $x$ and $p$ reflecting the photon number and phase fluctuations of the coherent state. Figure 16(b) presents the experimental Wigner function of the $n=3$ Fock state prepared by QND projective measurement, then reconstructed by our cavity QED-adapted tomographic procedure. It consists of a set of circular ripples centered at phase space origin. Its interfering features, displaying negative values, are distinctive of this field state "quantumness." The central symmetry of this Wigner function reveals that a Fock state has a complete phase indeterminacy.

\section{SCHRÖDINGER CAT STATES OF LIGHT AND DECOHERENCE STUDIES}

By describing how we count and manipulate photons in a cavity, I have so far emphasized the "particle aspect" of light. As recalled above, however, light is also a wave. Which of the particle or the wave aspect manifests itself depends upon the kind of experiment which is performed on the field. Let us describe now experiments in which the wave features of the field stored in the cavity is essential. This will lead to the description of photonic Schrödinger cats and to decoherence experiments.

At this stage, it is appropriate to recall Schrödinger's thought experiment (Schrödinger, 1935). The Austrian physicist imagined that a large system, a cat for instance, was coupled to a single atom, initially prepared in an excited state spontaneously decaying into a ground state by emitting a photon (or a radioactive particle). This emission was triggering a lethal device killing the cat. After half the lifetime of the excited state, the atom has evolved into a superposition of two states, one of which would be associated with the dead cat and the other to the live cat (Fig. 17). At this point, the atom and the cat would be entangled and the cat suspended between life and death. In our version of this experiment, we have a single atom in a superposition of two states and this atom controls the fate of a coherent field containing several photons (our Schrödinger cat) which takes two different phases at once, one that we can call "alive" and the other "dead." The way to perform this experiment was initially proposed in a paper written in 1991 together with our Brazilian colleagues Luiz Davidovich and Nicim Zagury (Brune et al., 1992). A similar proposal for the preparation of Schrödinger cat states of light in the optical domain had been made earlier in another context (Savage, Braunstein, and Walls, 1990).

Our method employs again the Ramsey interferometer. It starts with the preparation of a coherent field in the cavity, whose Wigner function is a Gaussian [Fig. 16(a)]. A single nonresonant atom is then prepared in a coherent superposition of two states, an atomic Schrödinger kitten, as I have already called it. This atom crosses the cavity and each of its two components shifts the phase of the field in opposite direction by a simple dispersive index effect. Here again, we take advantage of the huge coupling of Rydberg atoms to microwaves which makes a single atom index large enough to have a macroscopic effect on the field phase. At the cavity exit, the atom and the field are entangled, each atomic state being correlated to a field state with a different phase (the phase difference being close to $135^{\circ}$ in a typical experiment). We can consider that the field with its Gaussian Wigner distribution centered at a point in phase space is a meter used to measure the atom's energy. After the atom has been exposed to the second Ramsey pulse and detected, there is no way to know in which state the atom crossed the cavity and the field collapses into a Schrödinger cat superposition. In other words, the atomic Schrödinger kitten has produced a photonic Schrödinger cat which contains several photons on average.

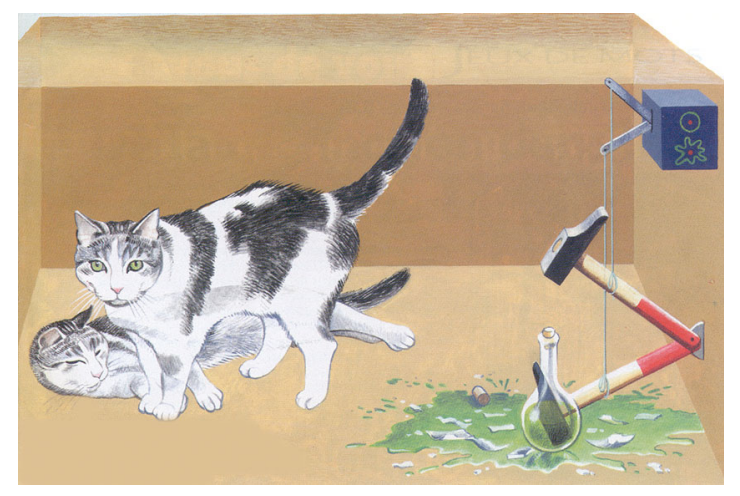

FIG. 17 (color). The Schrödinger cat thought experiment. From "Science et Vie Junior." 

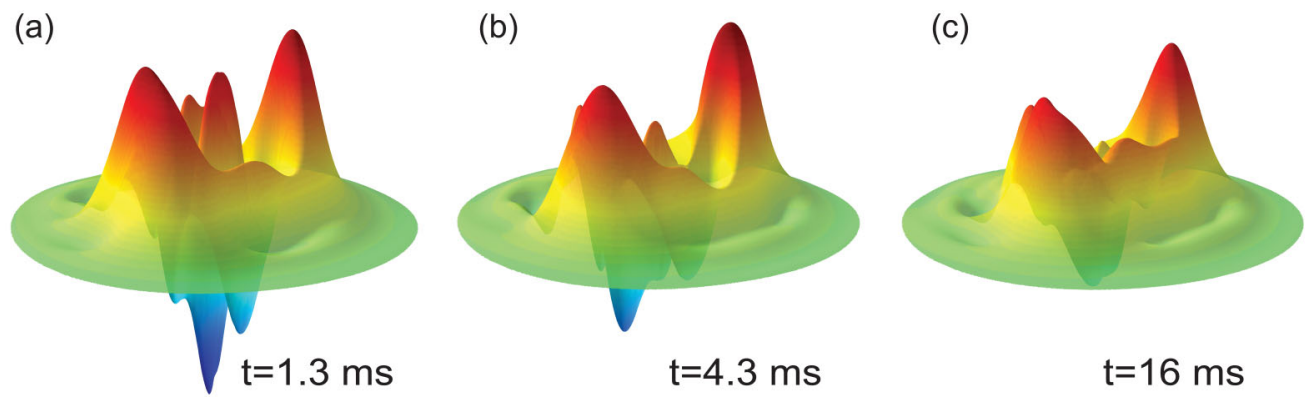

FIG. 18 (color). Schrödinger cat and decoherence: (a) Reconstruction of the Wigner function of a cat state with $n=3.5$ photons on average, a short time $(1.3 \mathrm{~ms})$ after its preparation by a single atom crossing the cavity. (b), (c) The same cat state after 4.3 and 16 ms: the vanishing of the fringe interfering features is a manifestation of decoherence. From Haroche, Brune, and Raimond, 2013.

By sending subsequent atoms across the cavity and achieving a tomographic field reconstruction with QND photon counting, we have been able in 2008 to reconstruct completely the Schrödinger cat state Wigner function (Deléglise et al., 2008). Figure 18(a) exhibits clearly the two peaks corresponding to the two classical fields of the superposition. The fringes between these peaks account for the coherence of the superposition. The negative values of these fringes is a signature of the quantumness of the "cat" state. Theory shows that the coupling of the field to the environment very quickly washes out the quantum coherence of the cat, leading it into a mundane statistical mixture of states. Wojciech Zurek has played an important role to elucidate the role of the environment in this process which occurs faster and faster as the "size" of the cat, measured by the square of the distance of its components in phase space, is increased (Zurek, 1991). For a given phase difference between the Gaussian components, this size is proportional to the cat's mean photon number.

We have studied this decoherence phenomenon by reconstructing the field Wigner function at various times (Deléglise et al., 2008). Figures 18(b) and 18(c), which represent snapshots of the Wigner function taken at increasing times after the Schrödinger cat state preparation, show decoherence in action. Within a time much shorter than the energy damping time of $130 \mathrm{~ms}$, the interfering features of the cat state are indeed suppressed, leaving the Wigner function as a sum of two quasi-Gaussian peaks. We have checked that decoherence occurs at a rate proportional to the size of the cat. It is important to stress that these Schrödinger cat state recordings, as all field state reconstructions, are obtained from analyzing many realizations of the experiment and performing complex statistical analysis of the data (Deléglise et al., 2008). Acquiring knowledge about a quantum state always requires such a statistical procedure and these experiments rely on the fact that we can prepare an arbitrary number of copies of the state to be reconstructed and follow the subsequent evolution of all these copies.

An earlier version of this experiment had been performed in 1996, with a cavity having a much shorter damping time, in $100 \mu$ s range (Brune, Hagley et al., 1996). Since it was not possible to send a sequence of measuring atoms across the cavity before its field had decayed, the experiment relied on the information provided by a single probe atom following the atom which had prepared the cat state. Instead of reconstructing the whole Wigner function, we used this single probe atom to get information about the Wigner function at phase space origin, where its value is very sensitive to the cat's coherence. Comparing the detection signal of the first atom which prepared the cat and that of the second which probed its coherence provided a two-atom correlation signal whose decay as a function of the delay between the two atoms measured the loss of quantum coherence of the cat versus time. Figure 19 shows this decay, for two cat states with different separations between their two components (as shown in the insets). The shortening of the decoherence time as the separation is increased was clearly demonstrated.

We have also prepared Schrödinger cat states of radiation by resonant atom-field interaction (Auffeves et al., 2003; Meunier et al., 2005). Letting a coherent field evolve under its coupling with a Rydberg atom at resonance turns the atomfield system, after some time, into an entangled atom-field state superposition involving two coherent fields with opposite phases. The two components of this cat merge together at a later time. This effect of field phase splitting and recombination is related to the collapse and revival of the Rabi oscillation phenomenon (Eberly, Narozhny, and

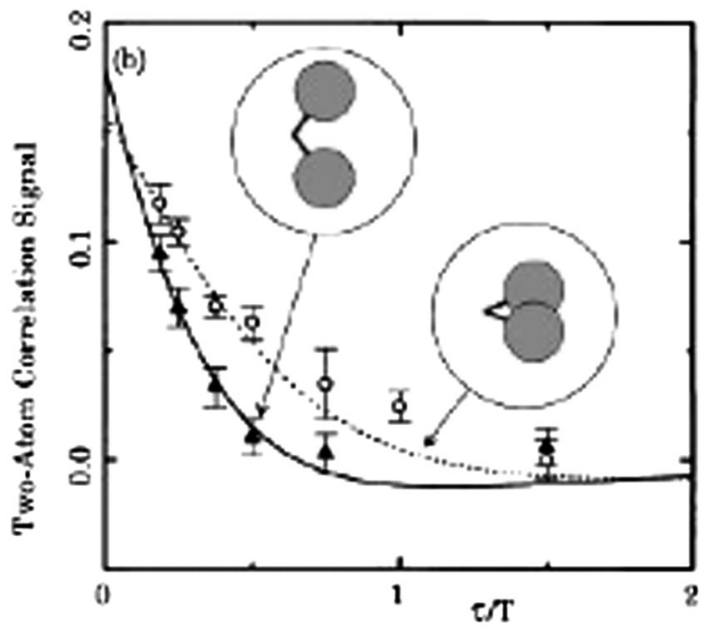

FIG. 19. Observation of decoherence in a two-atom experiment. The correlation signal is plotted vs the delay $\tau$ between the atom preparing the "cat state" and the atom probing its coherence. This delay is measured in units of the cavity damping time $T_{c}$ (equal to $160 \mu \mathrm{s}$ in this experiment). The points are experimental and the curves are theoretical. The two curves correspond to cat states with different separations between their components (as shown in the insets schematically representing their Gaussian components as circles). From Brune, Hagley et al., 1996. 
Sanchez-Mondragon, 1980). The Schrödinger cat experiments in cavity QED illustrate the fragility of quantum coherences in systems made of increasing numbers of particles. They give us a glimpse at the boundary between the quantum world, where state superpositions are ubiquitous, and the classical one, where systems behave in a mundane classical way. A detailed study of the cavity QED Schrödinger cats along with a review of proposals for the generation of various Schrödinger cats in quantum optics can be found in the book Exploring the Quantum: Atoms, Cavities and Photons (Haroche and Raimond, 2006). This book presents also a detailed analysis of the decoherence of these cat states.

\section{QUANTUM INFORMATION IN CAVITY QED}

The ENS photon trap and NIST ion-trap experiments are representative of a very broad field of experimental physics. The nondestructive control and manipulation of single quantum particles now performed in many laboratories around the world is strongly motivated by the prospect of exploiting these systems in order to develop new ways to process quantum information. This domain of research became very active in the mid 1990s, at the time we were building our photon box experiment, with the nondestructive photon counting and manipulation of light as our main goal. Feynman's reflections about quantum simulators (Feynman, 1985) and the propositions of cryptographic key distributions based on quantum laws (Bennett and Brassard, 1984; Ekert, 1991) date back to the 1980s and early 1990s but they did not have any resonance at that time on our work at ENS. Things changed with the proposal of quantum computers working with qubits made of atomic or light particles (Ekert and Josza, 1996; Nielsen and Chuang, 2000) and with the discovery of quantum algorithms which found an obvious use for such computers (Deutsch and Josza, 1992; Shor, 1994).

These ideas matured when our cavity QED setup became operational to test them, i.e., when the photon damping time in our open cavity reached a value (a couple hundred microseconds) exceeding the atom-cavity interaction time (about $20 \mu \mathrm{s}$ ). The convergence between these theoretical ideas and our experiments became then very fruitful. It was also around that time that we became influenced by the theoretical studies on decoherence (Zurek, 1991; Giulini et al., 1996) and the possibility to check them with simple experiments became for us a strong incentive. A review of the experimental methods we developed then to test basic steps of quantum information can be found in Raimond, Brune, and Haroche (2001).

While the QND photon counting and the Schrödinger cat state experiments described above are based on the dispersive nonresonant interaction of atoms with the cavity field, our cavity QED quantum information experiments have mostly exploited resonant atom-cavity field interactions. The basic phenomenon is then the Rabi oscillation, alluded to in the introduction of this Lecture. Figure 20 shows an experimental recording of the probability for finding the atom in its initial level $e$ as a function of its interaction time $t$ with the cavity initially in vacuum. This signal, observed in 1996 (Brune, Schmidt-Kaler et al., 1996), exhibits a damped oscillation at the vacuum Rabi frequency $\Omega_{0} / 2 \pi=50 \mathrm{kHz}$

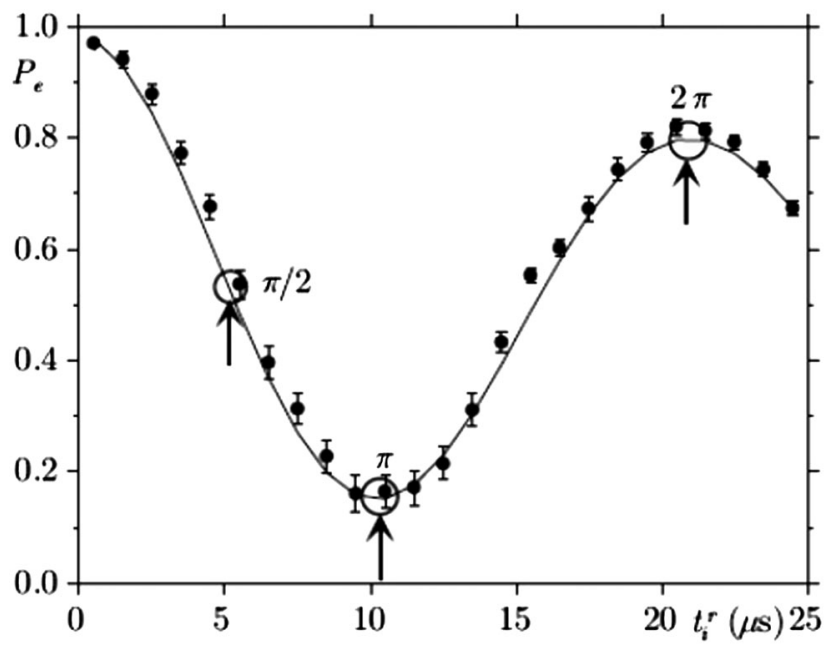

FIG. 20. Vacuum Rabi oscillation representing the probability for finding the atom in its initial state $e$ after interacting during time $t$ with the cavity initially in vacuum and resonant on the $e \rightarrow g$ transition. Points are experimental and the curve is a fit to a damped sinusoid. The three arrows mark the times corresponding to the $\pi / 2$, $\pi$, and $2 \pi$-Rabi pulses.

(the damping being due to various experimental imperfections). This oscillation is the direct manifestation of the strong coupling regime of cavity QED, whose observation had been our goal since the early 1980s. We reached it first in 1987 with the closed cylindrical cavity which we used for our two-photon Rydberg maser (Brune et al., 1987), then again in 1996 with our first open cavity able to keep a photon for a time longer than the atomic transit time across it.

It is only with this open cavity structure that we could easily vary the atom-cavity interaction time and thus directly record the Rabi oscillation. The method we use for this is to apply between the cavity mirrors an electric field which modifies by Stark effect the energies of the circular Rydberg states $e$ and $g$. This allows us to tune the atomic transition in and out of resonance at a determined time. The atoms are prepared in the circular state by a pulsed process which also fixes their velocity, so that we know when they enter the cavity and can decide when the Stark pulse must be applied to fix the atom-cavity coupling time (Raimond, Brune, and Haroche, 2001). Our quantum information experiments, performed in a setup similar to the one sketched in Fig. 10, were based on the exploitation of the Rabi oscillation in the main cavity $C$, combined with classical microwave pulses produced in the auxiliary cavities $R_{1}$ and $R_{2}$. These experiments took thus advantage in various ways of the versatile properties of the Ramsey interferometer setup.

Quantum information experiments manipulate two-level systems called qubits. In our case, these qubits are either atoms crossing the cavity while evolving between two Rydberg states or the cavity field oscillating between the two states $|0\rangle$ and $|1\rangle$ containing 0 and 1 photon. The coupling between the qubits is based on the realization of Rabi oscillation pulses of various durations. The auxiliary cavities $R_{1}$ and $R_{2}$ sandwiching the photon storing cavity $C$ are used to prepare and analyze the state of the atomic qubit, before or after their interaction with the cavity field. 
When the atom-field interaction time is set so that $\Omega_{0} t=$ $\pi / 2$, the Rabi oscillation maximally entangles the atom with the cavity field, realizing the transformation $|e, 0\rangle \rightarrow(|e 0\rangle+$ $|g, 1\rangle) / \sqrt{2}$. For $\Omega_{0} t=\pi$, the atom and the field exchange a quantum of excitation, realizing the transformations $|e, 0\rangle \rightarrow$ $|g, 1\rangle$ and $|g, 1\rangle \rightarrow-|e, 0\rangle$. Another useful Rabi pulse corresponds to $\Omega_{0} t=2 \pi$. It has the effect to induce a $\pi$-phase shift of the atom + field state, realizing the transformations $|e, 0\rangle \rightarrow-|e, 0\rangle$, and $|g, 1\rangle \rightarrow-|g, 1\rangle$, while the atom in state $|g\rangle$ crossing under the same conditions the cavity in vacuum is not affected: $|g, 0\rangle \rightarrow|g, 0\rangle$.

In order to maximally entangle two atoms, the first being prepared in state $e$ and the second in state $g$, we have sent them successively in a cavity initially in vacuum, realizing a $\pi / 2$-Rabi pulse on the first atom and a $\pi$-Rabi pulse on the second (Hagley et al., 1997). The first pulse entangles the first atom with the cavity field, while the second exchanges the second atom and the cavity field excitations, finally leading to the two-atom entangled state $(|e, g>-| g, e\rangle) / \sqrt{2}$. The cavity field, transiently entangled with the first atom, ends in its initial vacuum state, playing thus the role of a catalyst for the deterministic entanglement of the two atoms.

We have also realized a quantum memory, storing in the cavity the information initially carried by a first atom before copying it onto a second atom (Maître et al., 1997). The first atom prepared in a superposition state $a|e\rangle+b|g\rangle$ in $R_{1}$ undergoes a $\pi$-Rabi pulse in the initially empty cavity, preparing its field in the state $a|1\rangle+b|0\rangle$. A second atom initially in $|g\rangle$ then undergoes a $\pi$-Rabi pulse in this field, exiting the cavity in the state $-a|e\rangle+b|g\rangle$ which, within a phase shift, is identical to the initial state of the first atom. Applying to the second atom a classical microwave pulse in $R_{2}$ before detecting it allows us to analyze its final state, checking that the state superposition has been copied from the first atom to the second.

We have also demonstrated the operation of a quantum gate (Rauschenbeutel et al., 1999), an elementary device coupling a control and a target qubit in such a way that the target undergoes a unitary transformation if the control is in one state and remains unchanged if the control is in the other state (in all cases, the control state does not change). For this experiment, three circular atomic Rydberg states were relevant: the levels $e$ and $g$ with principal quantum numbers 51 and 50 (the transition between these states being resonant with the cavity mode) and a third circular Rydberg state with principal quantum number 49, called $i$ in the following. The transition $g$ to $i$ being far off resonant with the cavity, the atom in state $i$ was totally insensitive to the presence of photons in the cavity. The control qubit was then the cavity field in state 0 or 1 while the target qubit was a Rydberg atom evolving between the two states $g$ and $i$.

The interaction time between the atom and the cavity field was set to achieve a $2 \pi$-Rabi pulse in vacuum for an atom evolving between states $e$ and $g$. The coupling between the photon and atomic qubit then realized the mapping $|i, 0\rangle \rightarrow$ $|i, 0\rangle,|g, 0\rangle \rightarrow|g, 0\rangle, \quad|i, 1\rangle \rightarrow|i, 1\rangle$, and $|g, 1\rangle \rightarrow-|g, 1\rangle$. This is the "truth table" of a "phase" gate inducing a conditional $\pi$-phase shift onto the target qubit, if and only if the control is in the state 1. By applying to the target atomic qubit two pulses in $R_{1}$ and in $R_{2}$, equivalent to the so-called
Hadamard one qubit gates, this "phase gate" was transformed into a "control-not" gate leaving the target state unchanged if the control was in state 0 and switching the two target states if the control was in state 1 . Such a gate was operating as a $0-1$ photon QND counter since the final state of the atom crossing the cavity ( $i$ or $g$ ) was determined by the photon number in it ( 0 or 1$)$, which remained unchanged in the measurement process. This experiment realized in 1999 (Nogues et al., 1999) was a precursor of our later QND experiment (Gleyzes et al., 2007). It could not count photon numbers above 1 and could not repeat many times the counting procedure before the field decayed in the cavity.

The use of Rabi pulses of controlled duration acting on atoms crossing the cavity one by one can be described as a kind of deterministic quantum "knitting" procedure. By extending this procedure to three atoms, we have been able to prepare a triplet of entangled particles, a so-called GHZ state (Rauschenbeutel et al., 2000). The preparation involves a $\pi / 2$-Rabi pulse applied on a first atom, realizing an entangled first atom-cavity state, as described above. The state of the field is then read by applying a $2 \pi$-Rabi pulse on a second atom, realizing a QND counting of the photon number of the field left by the first atom in the cavity. The state of the two atoms and the field then becomes $(|e, 0, i\rangle+|g, 1, g\rangle) / \sqrt{2}$. A third atom, initially in $g$, then crosses the cavity and undergoes a $\pi$ pulse, thus copying the state of the field and bringing back the cavity in vacuum. The final three atom state is then $(|e, i, g\rangle+|g, g, e\rangle) / \sqrt{2}$ where the three symbols refer, in that order, to the three atoms having successively crossed the cavity.

In another entanglement experiment, we have tested the principle of complementarity by studying how the fringes of an atomic Ramsey interferometer vanish when information about the atomic path in the interferometer is stored in the field mixing the atomic levels (Bertet et al., 2001). Using again our Ramsey-cavity QED setup, we have also demonstrated how to exploit a cavity-assisted collision to entangle two atoms crossing simultaneously the field mode (Osnaghi et al. (2001) and how to entangle two field modes via their resonant interaction with a single atom (Rauschenbeutel et al., 2001). We have also shown how to exploit the dispersive interaction of atoms with the cavity field to detect atoms nondestructively by the phase shift they produce on a small coherent field (Maioli et al., 2005). All these experiments are described in detail in Haroche and Raimond (2006).

\section{PERSPECTIVES FOR THE ENS WORK}

In the course of our cavity QED studies we have learned how to measure quantum fields nondestructively, how to prepare and reconstruct various nonclassical states of radiation, and how to exploit the coupling of these fields to Rydberg atoms in order to demonstrate steps of quantum information procedures. We are presently developing these studies along several directions under a senior investigator grant obtained in 2009 from the European Research Council (ERC). The aim of this project is to push further the quantumclassical boundary by preparing nonclassical Fock and Schrödinger cat states of light containing larger numbers of photons, by studying the nonlocal properties of entangled 
fields stored in two cavities, and by investigating ways to protect efficiently nonclassical fields against decoherence.

Another line of research will consist of tailoring the Hamiltonian ruling the field evolution by exploiting the quantum Zeno effect. We have already demonstrated that it is possible to freeze the growth of a coherent field in the cavity by repetitive measurements of the field photon number (Bernu et al., 2008). With Italian colleagues, S. Pascazio and P. Facchi, we propose a generalization of this experiment in which we would use a repetitive QND procedure to find out whether the field contains a preset photon number $n_{0}$ in the cavity. By performing this measurement, we would prevent the field from reaching this number and force it to evolve in the subspaces spanned by Fock states with either $n<n_{0}$ or $n>n_{0}$. Restricting the evolution in this way would result in novel methods to generate photonic Schrödinger cats and more generally in new procedures to prepare and control arbitrary superpositions of coherent states in the cavity (Raimond et al., 2010, 2012). For these and other experiments, we need a setup in which atoms interact with the cavity field for times extending in the millisecond time range. An atomic-fountain setup in which slow atoms will cross the cavity at velocities of the order of a few meters per second is in preparation in order to achieve this condition. The atoms, cooled in a magneto-optical trap (Cohen-Tannoudji, 1998), will be pushed upward by a laser kick and sent in a superconducting cavity located above the trap at the turning point of the atomic parabolic trajectory. The slow atoms will be prepared into circular states and detected by field ionization inside the cavity.

\section{RELATION TO OTHER DOMAINS IN QUANTUM INFORMATION SCIENCE}

Our Rydberg atom-microwave superconducting cavity experiments are related to other experimental studies exploring, in different contexts, the atom-photon interaction in structures confining the electromagnetic field. Optical cavity QED has developed in parallel with microwave cavity QED and has reached the strong coupling regime in 1992 (Thompson, Rempe, and Kimble, 1992). In this very active domain of research, many experiments have been realized over the last 20 years to test quantum properties of light or to demonstrate basic operations in quantum information processing. These experiments often involve the interaction of cold atoms with optical photons and are exploiting the techniques of laser cooling and trapping of atoms in conventional or chip-based devices. They use various cavity designs including high finesse Fabry-Perot, microsphere, or microtorroidal structures sustaining whispering gallery modes. The diversity of studies is so large that giving extensive reference to specific works is beyond the scope of this lecture. A review of the early research in this domain can be found in Mabuchi and Doherty (2002) and a more recent description of the field is given in Rempe (2009). Other developments in solid state physics must be mentioned. There atoms are replaced by quantum dots embedded in Bragg layers or in photonic band gap materials confining the photons (Reithmaier, 2008). Many interesting cavity QED effects have been observed with these systems. Strong coupling of light emitters with microcavity structures has been developed to achieve operations useful for quantum communication and quantum information processing purposes. Here again, I will not attempt to give more references to specific works.

A special mention should be made to the field of circuit QED (Schoelkopf and Girvin, 2008) because of its close connection with our microwave cavity QED work. In this field which has undergone a very fast and spectacular development lately, atoms are replaced by small superconducting circuits with Josephson junctions which behave as two-level artificial atoms coupled to strip line, coplanar structures or three-dimensional closed cavities sustaining radio-frequency fields. The coupling between the artificial atoms and the field obeys the same Jaynes-Cummings Hamiltonian as our Rydberg atom-superconducting cavity system (Fink et al., 2008). Many groups in the world are working in this new field and experiments very similar to the ones performed at ENS have been made with these devices, including the generation and study of Fock (Schuster et al., 2007; Hofheinz et al., 2008) and photonic Schrödinger cat states (Hofheinz et al., 2009; Kirchmair et al., 2013). The very strong coupling of the giant artificial Josephson atoms to radio frequencies leads to a faster dynamics than that of the Rydberg atomsuperconducting cavity coupling. Quantum processing operations with these systems occur at the nanosecond time scale, instead of the microsecond time scale of the Rydberg atom experiments. These circuits can be produced by wellestablished lithographic methods which makes them promising for scalable quantum information applications, provided their decoherence could be better controlled.

The ion trappers, as the photon ones, also form a wide community. David Wineland, in his Lecture, mentions experiments carried on by many teams around the world with ions in traps, which bear strong similarities with his own work. In a broader quantum information context, it is also important to mention, beyond cavity QED and ion-trap physics, the wide variety of quantum optics experiments exploiting the detection of photon correlations produced by various sources of twinlight beams. These experiments started with the pioneering demonstrations of Bell's inequality violations in the 1970s and early 1980s (Freedman and Clauser, 1972; Aspect, Dalibard, and Roger, 1982). They have since then demonstrated various properties of nonclassical light and explored their possible applications for quantum communication (Gisin et al., 2002), quantum teleportation (Bouwmeester et al., 1997), and quantum computing (Walther et al., 2005). The quantum nondemolition studies of propagating optical beams (Grangier, Levenson, and Poizat, 1998) have not reached the single quantum sensitivity, but they nevertheless share some of their features with our QND work. Schrödinger cat states of light presenting many similarities with our cavity QED cats have also been generated in quantum optics experiments (Ourjoumtsev et al., 2007). The domain is again too vast to mention specifically more works. Experiments with cold atoms, especially those which study the properties of atoms trapped in optical lattices in one, two, or three dimensions, are also aiming at manipulating single particles in a controlled environment, with quantum information applications in mind (Garcia-Ripoli and Cirac, 2003; Negretti, Treutlein, and Calarco, 2011). Finally, the development of nanomechanical oscillators coupled to atomic systems or to electromagnetic 
field resonators opens a new domain where the manifestations of state superposition and entanglement will soon become observable (Rocheleau et al., 2009; Chan et al., 2011). Many of the concepts and ideas exploited in these experiments have features in common with those used in cavity QED or ion-trap physics.

Without comparing the respective merits or limitations of the various lines of research aiming at controlling quantum systems, we can try to foresee the future of this physics at large. The all purpose quantum computer which would be able to implement arbitrary algorithms seems to be still very much an utopia (Haroche and Raimond, 1996). The problem of decoherence is indeed extremely difficult to solve in spite of the progress made in the implementation of error correction (Schindler et al., 2011; Reed et al., 2012). Quantum simulators emulating condensed matter structures and dynamics with arrays of atoms in optical lattices (Jaksch and Zoller, 2005) or ions in traps (Schneider, Porras, and Schaetz, 2012) seem more realistic. They will probably lead to important advances in the near future. Quantum communication with photons and the realization of quantum nodes in which information will be exchanged between atomic systems and photons in order to build efficient quantum repeaters are also likely to be developed (Kimble, 2008). The ideas of cavity QED seem to be particularly well adapted in this context. Important progresses in quantum metrology (Giovannetti, Lloyd, and Maccone, 2011) using the tools of particle control can also be expected. But I am well aware that this exercise in prediction is largely futile. The most important applications of quantum physics have come in the past from the serendipitous convergence of disparate blue sky advances made by scientists who would never have predicted what their results were going to lead to. I guess the same will happen with the control of single quantum particles.

\section{ACKNOWLEDGMENTS}

Let me add a few more personal remarks. The work accomplished in my laboratory during the last three decades has been a collective endeavor in which my former students and now colleagues Jean-Michel Raimond and Michel Brune have played an essential role. I have been extremely lucky that we have stayed together over all these years and that we have been able to build such a strong and friendly collaboration (Fig. 21 shows us in the lab, in the autumn of 2012). We have always discussed openly and freely our projects and decided together the way to follow. Many of the seminal ideas in our work have come from Jean-Michel or Michel and I am well aware that today's recognition is theirs as well as mine. I know they share this feeling! This work has involved many people, almost a hundred students, postdocs, and visitors altogether. The last generation is shown in the photograph of Fig. 22 taken in October 2012, three days after the Nobel Prize was announced. Besides Jean-Michel and Michel, I would like to mention two young colleagues present in this photograph, Sebastien Gleyzes and Igor Dotsenko, whose contribution to the photon detection, Schrödinger cat, and quantum feedback experiments has been very important. Stefan Kuhr (now at the University of Strathclyde in Glasgow) was a postdoc in our group when we realized the first QND photon counting in 2006 and his contribution too

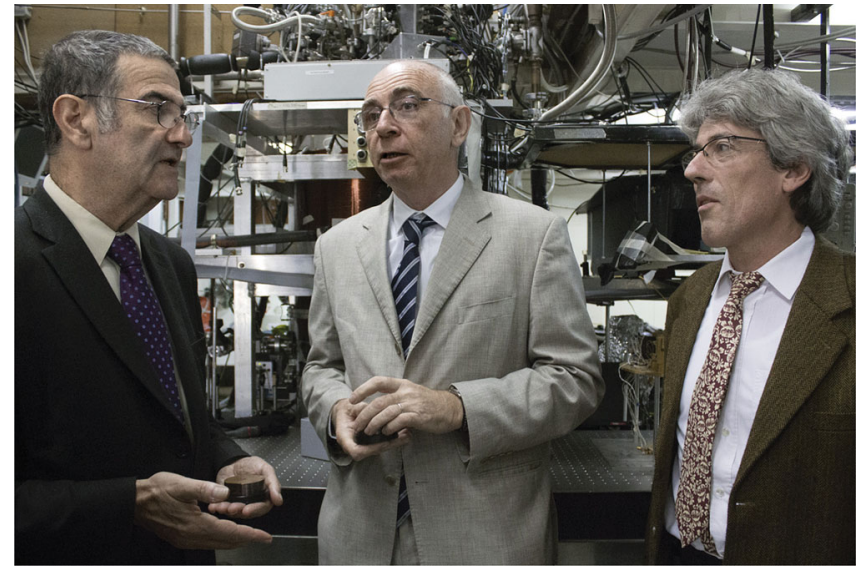

FIG. 21 (color). The author, Jean-Michel Raimond, and Michel Brune (from left to right) in the lab in autumn 2012 (credit J. P. Dars, Photothèque de l'ENS).

has been essential. Gilles Nogues, after having been a graduate student, has accompanied us until 2010 before taking up a position in Grenoble. Among the important participants to our work in earlier stages, I must recall the role of Philippe Goy whose technological skills and deep insight in physics has been essential. Michel Gross and Jean Hare played an important role to develop our circular Rydberg atom preparation procedure. Valerie Lefevre-Seguin was a member of our group when our QND ideas matured and Ferdinand Schmidt-Kaler, now in Mainz, helped us to realize the first experiments in the strong coupling regime with open cavities. On the theory side, the fruitful collaboration with our Brazilian colleagues Luiz Davidovich and Nicim Zagury has also been important. Other students, postdocs, visitors, and collaborators are listed as co-authors in the references given at the end of this paper. I hope they will forgive me not mentioning them here by name. All have by their successive contributions improved step by step our expertise and cleverness in our photon juggling game. Coming from many

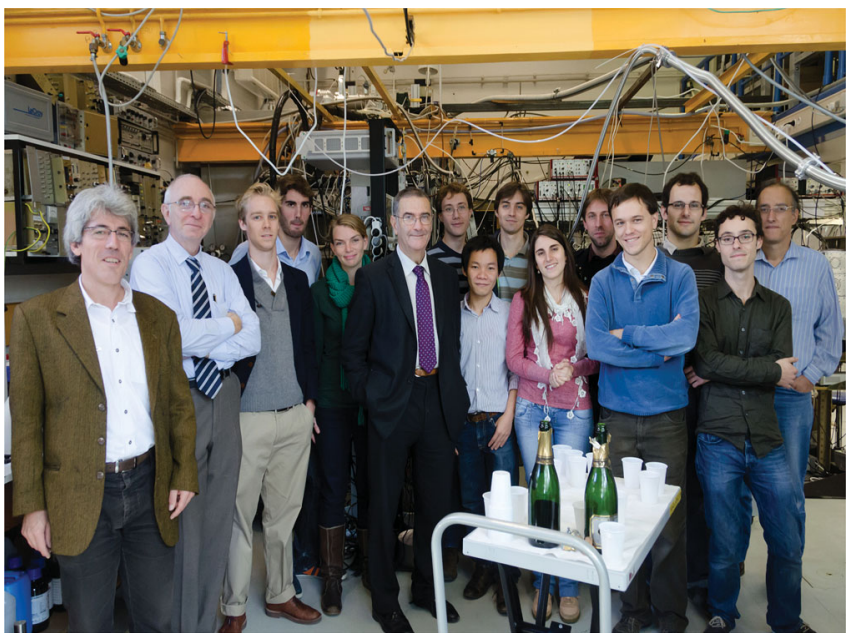

FIG. 22 (color). The ENS team in October 2012: from left to right: M. Brune, J.-M. Raimond, A. Facon, A. Signoles, E.-K. Dietsche, S. Haroche, T. Rybarczyk, Nguyen Thanh Long, I. Dotsenko, C. Herman, S. Guerlich, B. Peaudecerf, S. Gleyzes, R. Texeira, and J. Hare (a colleague, former group member). 


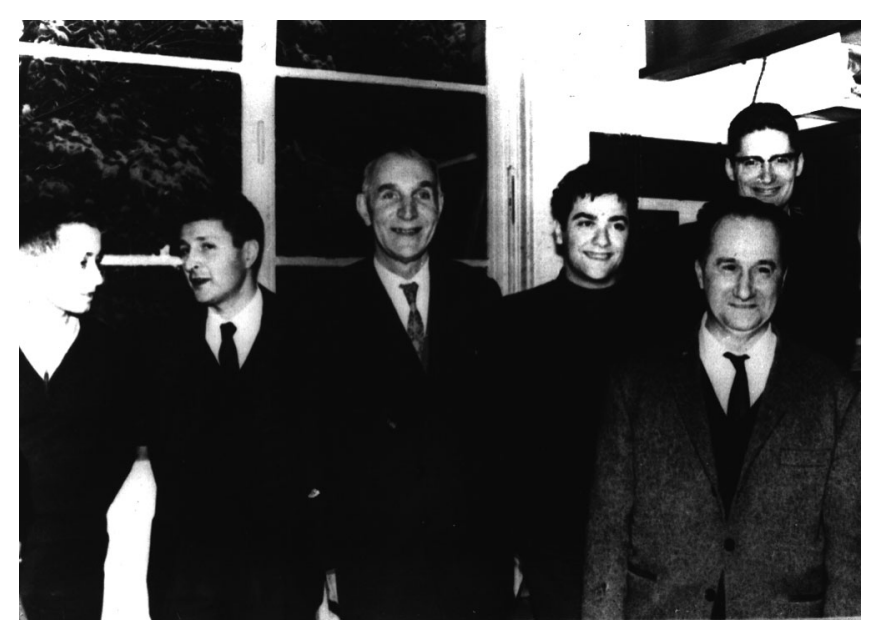

FIG. 23. Photograph taken on the day of the Alfred Kastler Nobel Prize announcement, in the same room as the photograph in Fig. 22. From left to right: Franck Laloë, Claude Cohen-Tannoudji (Nobel 1997), Alfred Kastler (Nobel 1966), Serge Haroche, Jean Brossel, and Alain Omont.

countries, they have been working hard and with an enthusiasm that the inevitable drawbacks of an experimenter's life could not diminish. I will always remember some exhilarating moments when our efforts were rewarded and the difficulties forgotten: the 1987 Bastille day (or rather night) in the lab when we observed with our friend Luiz Davidovich the first evidence that our two-photon micromaser was working; the early morning in a Sydney hotel in July 1996 when I received by fax, for immediate presentation in the talk I was going to deliver at a conference, the long awaited Schrödinger cat signals that Michel, Jean-Michel, and the students had just obtained while I was sleeping $20000 \mathrm{~km}$ and ten time zones away; and the evening of 11 September 2006 (my birthday!) when Stefan Kuhr summoned me back by phone to the lab, just in time to observe the first recording of the birth and death of a single photon in our cavity. I must also recall the unique context of the Kastler Brossel Laboratory, in which we have been working all these years in an atmosphere of trust, feeling always free to follow our ideas without having to justify our work by promising improbable applications. This spirit of freedom is the inheritance of great precursors, Alfred Kastler and Jean Brossel, who built this lab at the time they invented optical pumping, 60 years ago. Claude CohenTannoudji followed their steps and infused me with his passion for the quantum world. The photograph of Fig. 23 was taken at ENS, in the same room as the previous one, 46 years almost to the day before, when the Nobel Prize of Alfred Kastler was announced. Claude and I have certainly changed physically, but our enthusiasm for physics has remained the same! At the start of my career, I was funded directly by the laboratory, without ever being asked to write proposals or lengthy reports. Things changed progressively, when research became more project oriented at the end of the 1980s. My group and its work were then recognized well enough to get grants from the CNRS and the Agence Nationale de la Recherche, as well as from European agencies, culminating with our ERC senior grant in 2009. I must also mention a generous funding by the Japanese Science and Technology Corporation (JST) through a collaborative grant that I shared with Yoshisha Yamamoto from 1999 to 2009.
It allowed us to pursue freely our research on quantum entanglement without any bureaucratic red tape. I would not be complete without stressing that teaching has always been an important stimulation for me. At the University Paris VI and at ENS, I have found that preparing lectures and interacting with students has greatly helped me to understand physics better and has more than once given me new ideas for experiments. Since 2001 at College de France, I have had the challenging task to teach a new course each year about topics in quantum physics directly related to my own research or to that of colleagues working in quantum information science. This experience has also been extraordinarily rewarding and fruitful. Let me also acknowledge the influence on my work of so many friends and colleagues from all over the world. I have often been stimulated by attending their lectures, reading about their work, or discussing with them in Paris or in their own laboratory. These friendships are the privilege of our lives as scientists. I cherish the opportunity we have to exchange with people of many different cultures sharing, in our diversity, the same curiosity and the same passion for discovering and understanding nature. My old friendship with David Wineland is especially worth mentioning here, since it has been a special pleasure for me to share this Nobel prize with him. Finally nothing would have been possible without Claudine, my wife whom I met long before knowing what a photon was, and Julien and Judith, our children. They have illuminated my life with their love, their humor, and their intellectual support. But this part of the story will be told elsewhere (Haroche, 2013).

\section{REFERENCES}

Aspect, A., J. Dalibard, and G. Roger, 1982, Phys. Rev. Lett. 49, 1804.

Auffeves, A., P. Maioli, T. Meunier, S. Gleyzes, G. Nogues, M. Brune, J.-M. Raimond, and S. Haroche, 2003, Phys. Rev. Lett. 91, 230405

Bennett, C. H., and G. Brassard, 1984, in Proceedings of the IEEE International Conference on Computers, Systems and Signal Processing (IEEE, New York), p 175.

Bernu, J., S. Deléglise, C. Sayrin, S. Kuhr, I. Dotsenko, M. Brune, J.-M. Raimond, and S. Haroche, 2008, Phys. Rev. Lett. 101, 180402.

Bertet, P., S. Osnaghi, A. Rauschenbeutel, G. Nogues, A. Auffeves, M. Brune, J.-M. Raimond, and S. Haroche, 2001, Nature (London) 411, 166.

Bohr, N., 1949, Discussion with Einstein on epistemological problems in atomic physics, in Albert Einstein, Philosopher-scientist, edited by Paul Arthur Shilpp (Harper, New York).

Bonifacio, R., P. Schwendimann, and F. Haake, 1971, Phys. Rev. A 4, 302.

Bouwmeester, D., J. M. Pan, K. Mattle, M. Eibl, H. Weinfurter, and A. Zeilinger, 1997, Nature (London) 390, 575.

Braginsky, V. B., and F. Y. Khalili, 1996, Rev. Mod. Phys. 68, 1.

Braginsky, V. B., Y. I. Vorontosov, and F. Y. Khalili, 1977, Zh. Eksp. Teor. Fiz. 73, 1340 [Sov. Phys. JETP 46, 705 (1977)].

Braginsky, V.B., and S.P. Vyatchanin, 1981, Dokl. Akad. Nauk SSSR 259, 570 [Sov. Phys. Dokl. 26, 686 (1981)].

Brune, M., J. Bernu, C. Guerlin, S. Deléglise, C. Sayrin, S. Gleyzes, S. Kuhr, I. Dotsenko, J.-M. Raimond, and S. Haroche, 2008, Phys. Rev. Lett. 101, 240402.

Brune, M., E. Hagley, J. Dreyer, X. Maïtre, A. Maali, C. Wunderlich, J.-M. Raimond, and S. Haroche, 1996, Phys. Rev. Lett. 77, 4887. 
Brune, M., S. Haroche, V. Lefevre-Seguin, J.-M. Raimond, and N. Zagury, 1990, Phys. Rev. Lett. 65, 976.

Brune, M., S. Haroche, J.-M. Raimond, L. Davidovich, and N. Zagury, 1992, Phys. Rev. A 45, 5193.

Brune, M., J.-M. Raimond, P. Goy, L. Davidovich, and S. Haroche, 1987, Phys. Rev. Lett. 59, 1899.

Brune, M., F. Schmidt-Kaler, A. Maali, J. Dreyer, E. Hagley, J.-M. Raimond, and S. Haroche, 1996, Phys. Rev. Lett. 76, 1800.

Chan, J., T. P. M. Alegre, A. H. Safavi-Naeini, J. T. Hill, A. Krause, S. Groblacher, M. Aspelmeyer, and O. Painter, 2011, Nature (London) 478, 89.

Chu, S., 1998, Rev. Mod. Phys. 70, 685.

Cohen-Tannoudji, C., 1962a, Ann. Phys. (Paris) 7, 423.

Cohen-Tannoudji, C., 1962b, Ann. Phys. (Paris) 7, 469.

Cohen-Tannoudji, C., 1998, Rev. Mod. Phys. 70, 707.

Cohen-Tannoudji, C., and S. Haroche, 1965, C. R. Acad. Sci., Paris 261, 5400 .

Cohen-Tannoudji, C., and S. Haroche, 1969, J. Phys. France 30, 153.

Cohen-Tannoudji, C., S. Haroche, C. Audoin, and J.-P. Schermann, 1970, Phys. Rev. Lett. 24, 861.

Deléglise, S., I. Dotsenko, C. Sayrin, J. Bernu, M. Brune, J.-M. Raimond, and S. Haroche, 2008, Nature (London) 455, 510 .

Deutsch, D., and R. Josza, 1992, Proc. R. Soc. A 439, 553.

Dicke, R. H., 1954, Phys. Rev. 93, 99.

Dotsenko, I., M. Mirrahimi, M. Brune, S. Haroche, J.-M. Raimond, and P. Rouchon, 2009, Phys. Rev. A 80, 013805.

Dukas, T. W., M. G. Littman, R. R. Freeman, and D. Kleppner, 1975, Phys. Rev. Lett. 35, 366.

Eberly, J. H., N. B. Narozhny, and J. J. Sanchez-Mondragon, 1980, Phys. Rev. Lett. 44, 1323.

Ekert, A., 1991, Phys. Rev. Lett. 67, 661.

Ekert, A., and R. Josza, 1996, Rev. Mod. Phys. 68, 733.

Fabre, C., P. Goy, and S. Haroche, 1977, J. Phys. B. Letters 10, L-183.

Fabre, C., P. Goy, and S. Haroche, 1978, Phys. Rev. A 18, 229.

Feynman, R., 1985, Opt. News 11, 11.

Fink, J. M., M. Göppl, M. Baur, R. Bianchetti, P. J. Leek, A. Blais, and A. Wallraff, 2008, Nature (London) 454, 315.

Freedman, S. J., and J. F. Clauser, 1972, Phys. Rev. Lett. 28, 938.

Gabor, D., 1971, Holography 1948-1971, Nobel Lecture on Nobelprize.org.

Gabrielse, G., and H. Dehmelt, 1985, Phys. Rev. Lett. 55, 67.

Garcia-Ripoli, J. J., and J. I. Cirac, 2003, Phil. Trans. R. Soc. A 361, 1537.

Giovannetti, V., S. Lloyd, and L. Maccone, 2011, Nat. Photonics 5, 222.

Gisin, N., G. G. Ribordy, W. Tittel, and H. Zbinden, 2002, Rev. Mod. Phys. 74, 145.

Giulini, D., E. Joos, C. Kiefer, J. Kupsch, I. O. Stamatescu, and H. D. Zeh, 1996, Decoherence and the Appearance of a Classical World in Quantum Theory (Springer, Berlin).

Glauber, R. J., 1963, Phys. Rev. 131, 2766.

Gleyzes, S., S. Kuhr, C. Guerlin, J. Bernu, S. Deleglise, U. Busk Hoff, M. Brune, J.-M. Raimond, and S. Haroche, 2007, Nature (London) 446, 297.

Goy, P., J.-M. Raimond, M. Gross, and S. Haroche, 1983, Phys. Rev. Lett. 50, 1903.

Grangier, P., J. A. Levenson, and J. P. Poizat, 1998, Nature (London) 396, 537.

Gross, M., P. Goy, S. Haroche, C. Fabre, and J.-M. Raimond, 1979, Phys. Rev. Lett. 43, 343.

Gross, M., and S. Haroche, 1982, Phys. Rep. 93, 301.
Guerlin, C., J. Bernu, S. Deléglise, C. Sayrin, S. Gleyzes, S. Kuhr, M. Brune, J.-M. Raimond, and S. Haroche, 2007, Nature (London) 448, 889.

Hagley, E., X. Maïtre, G. Nogues, C. Wunderlich, M. Brune, J.-M. Raimond, and S. Haroche, 1997, Phys. Rev. Lett. 79, 1.

Haroche, S., 1971a, Ann. Phys. (Paris) 6, 189.

Haroche, S., 1971b, Ann. Phys. (Paris) 6, 327.

Haroche, S., 1984, in Les Houches Summer School Lecture Notes, edited by G. Grynberg, and R. Stora (North-Holland, Amsterdam).

Haroche, S., 2013, Autobiography, to be posted on Nobelprize.org.

Haroche, S., M. Brune, and J.-M. Raimond, 2013, Phys. Today 66, No. 1, 27.

Haroche, S., and C. Cohen-Tannoudji, 1969, J. Phys. (France) 30, 125.

Haroche, S., M. Gross, and M. Silverman, 1974, Phys. Rev. Lett. 33, 1063.

Haroche, S., and D. Kleppner, 1989, Phys. Today 42, No. 1, 24.

Haroche, S., J. Paisner, and A. L. Schawlow, 1973, Phys. Rev. Lett. 30, 948.

Haroche, S. and J.-M. Raimond, 1996, Phys. Today 49, No. 8, 51.

Haroche, S., and J.-M. Raimond, 2006, Exploring the Quantum: Atoms, Cavities and Photons (Oxford University Press, Oxford, England).

Hofheinz, M., E. M. Weig, M. Ansman, R. C. Bialczak, E. Lucero, M. Neeley, A. D. O'Connell, H. Wang, J. M. Martinis, and A. N. Cleland, 2008, Nature (London) 454, 310.

Hofheinz, M., E. M. Weig, M. Ansman, R. C. Bialczak, E. Lucero, M. Neeley, A. D. O'Connell, D. Sank, J. Wenner, J. M. Martinis, and A. N. Cleland, 2009, Nature (London) 459, 546.

Hulet, R. G., E. S. Hilfer, and D. Kleppner, 1985, Phys. Rev. Lett. 55, 2137.

Hulet, R. G., and D. Kleppner, 1983, Phys. Rev. Lett. 51, 1430.

Jaksch, D., and P. Zoller, 2005, Ann. Phys. (Amsterdam) 315, 52.

Jaynes, E. T., and F. W. Cummings, 1963, Proc. IEEE 51, 89.

Jhe, W., A. Anderson, E. Hinds, D. Meschede, L. Moi, and S. Haroche, 1987, Phys. Rev. Lett. 58, 666.

Kaluzny, Y., P. Goy, M. Gross, J.-M. Raimond, and S. Haroche, 1983, Phys. Rev. Lett. 51, 1175.

Kimble, H. J., 2008, Nature (London) 453, 1023.

Kirchmair, G., B. Vlastakis, Z. Leghtas, S. E. Nigg, H. Paik, E. Ginossar, M. Mirrahimi, L. Frunzio, S. M. Girvin, and R. J. Schoelkopf, 2013, Nature (London) 495, 205.

Kleppner, D., 1981, Phys. Rev. Lett. 47, 233.

Kuhr, S., S. Gleyzes, C. Guerlin, J. Bernu, U-B. Hoff, S. Deléglise, S. Osnaghi, M. Brune, J-M. Raimond, S. Haroche, E. Jacques, P. Bosland, and B. Visentin, 2007, Appl. Phys. Lett. 90, 164101.

Mabuchi, H., and A.C. Doherty, 2002, Science 298, 1372.

Maioli, P., T. Meunier, S. Gleyzes, A. Auffeves, G. Nogues, M. Brune, J.-M. Raimond, and S. Haroche, 2005, Phys. Rev. Lett. 94, 113601.

Maître, X., E. Hagley, G. Nogues, C. Wunderlich, P. Goy, M. Brune, J.-M. Raimond, and S. Haroche, 1997, Phys. Rev. Lett. 79, 769.

Meekhof, D. M., C. Monroe, B.E. King, W. M. Itano, and D. J. Wineland, 1996, Phys. Rev. Lett. 76, 1796.

Meschede, D., H. Walther, and G. Muller, 1985, Phys. Rev. Lett. 54, 551.

Meunier, T., S. Gleyzes, P. Maioli, A. Auffeves, G. Nogues, M. Brune, J.-M. Raimond, and S. Haroche, 2005, Phys. Rev. Lett. 94, 010401.

Meystre, P., G. Rempe, and H. Walther, 1988, Opt. Lett. 13, 1078. Negretti, A., P. Treutlein, and T. Calarco, 2011, Quantum Inf. Process. 10, 721.

Nielsen, M. A., and I. L. Chuang, 2000, Quantum Computation and Quantum Information (Cambridge University Press, Cambridge, England). 
Nikolaus, B., D. Z. Zangh, and P. Toscheck, 1981, Phys. Rev. Lett. 47, 171

Nogues, G., A. Rauschenbeutel, S. Osnaghi, M. Brune, J.-M. Raimond, and S. Haroche, 1999, Nature (London) 400, 239.

Nussenzveig, P., F. Bernardot, M. Brune, J. Hare, J.-M. Raimond, S.

Haroche, and W. Gawlik, 1993, Phys. Rev. A 48, 3991.

Osnaghi, S., P. Bertet, A. Auffeves, P. Maioli, M. Brune, J.-M. Raimond, and S. Haroche, 2001, Phys. Rev. Lett. 87, 037902.

Ourjoumtsev, A., H. Jeong, R. Tualle-Brouri, and P. Grangier, 2007, Nature (London) 448, 784.

Peil, S., and G. Gabrielse, 1999, Phys. Rev. Lett. 83, 1287.

Phillips, W. D., 1998, Rev. Mod. Phys. 70, 721.

Purcell, E. M., 1946, Phys. Rev. 69, 681.

Raimond, J.-M., M. Brune, and S. Haroche, 2001, Rev. Mod. Phys. 73, 565.

Raimond, J.-M., P. Facchi, B. Peaudecerf, S. Pascazio, C. Sayrin, I. Dotsenko, S. Gleyzes, M. Brune, and S. Haroche, 2012, Phys. Rev. A 86, 032120.

Raimond, J.-M., P. Goy, M. Gross, C. Fabre, and S. Haroche, 1982a, Phys. Rev. Lett. 49, 1924.

Raimond, J.-M., P. Goy, M. Gross, C. Fabre, and S. Haroche, 1982b, Phys. Rev. Lett. 49, 117.

Raimond, J.-M., C. Sayrin, S. Gleyzes, I. Dotsenko, M. Brune, S. Haroche, P. Facchi, and S. Pascazio, 2010, Phys. Rev. Lett. 105, 213601.

Ramsey, N. F., 1949, Phys. Rev. 76, 996.

Rauschenbeutel, A., P. Bertet, S. Osnaghi, G. Nogues, M. Brune, J.-M. Raimond, and S. Haroche, 2001, Phys. Rev. A 64, 050301(R).

Rauschenbeutel, A., G. Nogues, S. Osnaghi, P. Bertet, M. Brune, J.-M. Raimond, and S. Haroche, 1999, Phys. Rev. Lett. 83, 5166.

Rauschenbeutel, A., G. Nogues, S. Osnaghi, P. Bertet, M. Brune, J.-M. Raimond, and S. Haroche, 2000, Science 288, 2024.

Reed, M.D., L. DiCarlo, S.E. Nigg, L. Sun, L. Frunzio, S. M. Girvin, and R. J. Schoelkopf, 2012, Nature (London) 482, 382.

Reithmaier, J. P., 2008, Semicond. Sci. Technol. 23, 123001.

Rempe, G., 2009, in Proceedings of Lasers and Electro-optics 2009 and the European Quantum Electronics Conference CLEO Europe-EQEC (IEEEXplore).
Rempe, G., F. Schmidt-Kaler, and H. Walther, 1990, Phys. Rev. Lett. 64, 2783.

Rocheleau, T., T. Ndukum, C. Macklin, J. B. Hertzberg, A. A. Clerk, and K. C. Schwab, 2009, Nature (London) 463, 72.

Savage, C. M., S. L. Braunstein, and D. F. Walls, 1990, Opt. Lett. 15, 628.

Sayrin, C., I. Dotsenko, X.X. Zhou, B. Peaudecerf, T. Rybarczyk, S. Gleyzes, P. Rouchon, M. Mirrahimi, H. Amini, M. Brune, J-M. Raimond, and S. Haroche, 2011, Nature (London) 477, 73.

Schindler, P., J. T. Barreiro, T. Monz, V. Nebendahl, D. Nigg, M. Chwalla, M. Hennrich, and R. Blatt, 2011, Science 332, 1059.

Schleich, W., 2005, Quantum Optics in Phase Space (Wiley, New York).

Schneider, C., D. Porras, and T. Schaetz, 2012, Rep. Prog. Phys. 75, 024401.

Schoelkopf, R. J., and S. M. Girvin, 2008, Nature (London) 451, 664. Schrödinger, E., 1935, Naturwissenschaften 23, 844.

Schrödinger, E., 1952, Brit. J. Philos. Sci. 3, 233.

Schuster, D. I., A. A. Houck, J. A. Schreier, A. Wallraff, J.M. Gambetta, A. Blais, L. Frunzio, J. Majer, B. Johnson, M. Devoret, S. M. Girvin, and R. J. Schoelkopf, 2007, Nature (London) 445, 515.

Scully, M. O., B.-G. Englert, and H. Walther, 1991, Nature (London) 351, 111.

Shor, P. W., 1994, in Proceedings of the 35th Annual Symposium on the Foundations of Computer Science (IEEE Computer Society Press, Los Alamitos, CA), p. 124.

Smithey, D. T., M. Beck, and M. G. Raymer, 1993, Phys. Rev. Lett. 70, 1244.

Thompson, R.J., G. Rempe, and H.J. Kimble, 1992, Phys. Rev. Lett. 68, 1132.

Walther, P., K. J. Resch, T. Rudolph, E. Schenck, H. Weinfurter, V. Vedral, M. Aspelmeyer, and A. Zeilinger, 2005, Nature (London) 434, 169.

Zhou, X. X., I. Dotsenko, B. Peaudecerf, T. Rybarczyk, C. Sayrin, S. Gleyzes, J.-M. Raimond, M. Brune, and S. Haroche, 2012, Phys. Rev. Lett. 108, 243602.

Zurek, W.H., 1991, Phys. Today 44, No. 10, 36. 